ITEP-TH-23/98

RIMS-1198

\title{
Weyl group extension of quantized current algebras
}

\author{
Jintai Ding *円 and Sergei Khoroshkin ** \& \\ ${ }^{*}$ Research Institute for Mathematical Sciences, Kyoto University, Kyoto 606, Japan \\ *Institute of Theoretical $\&$ Experimental Physics, 117259 Moscow, Russia
}

\begin{abstract}
In this paper, we extend the Drinfeld current realization of quantum affine algebras $U_{q}(\hat{\mathbf{g}})$ and of the Yangians in several directions: we construct current operators for non-simple roots of $\mathbf{g}$, define a new braid group action in terms of the current operators and describe the universal R-matrix for the corresponding "Drinfeld" comultiplication in the form of infinite product and in the form of certain integrals over current operators.
\end{abstract}

\section{Introduction}

In the theory of simple Lie algebras, the theory of affine Lie algebras $\hat{\mathrm{g}}$ distinguishs themselves from others due to the realization of the affine Lie algebras in current operators (or loop realizations). The current operator is an operator define as $x(z)=\Sigma_{n \in \mathbf{Z}} x_{n} z^{-n}$, where $x_{n}$ is an operator in the algebra and $z$ is a formal variable. One can associate to each root of $\mathbf{g}$ a current operator $x_{\alpha}(z)$, which gives a complete description of the whole Lie algebra $\hat{\mathbf{g}}$. The advantage of the current realization of affine Lie algebras lies in the fact that the formal variable $z$, which we can also treated as a number in $\mathbf{C}$, allows us to connect the algebraic structures of affine Lie algebra with analytic, geometric and topological structures, which is manifest best in the theory of Knizhinik-Zamolochikov equations. Current realization of quantum affine algebras was first given by Drinfeld after a few years of the discovery of the theory of quantum groups [Dr2]. The connection of the Drinfeld realization with the first realization of quantum groups given by Jimbo and Drinfeld and with other type of realizations is also highly nontrivial [Be] [KT2] [KT3] [DF]. Drinfeld current realization of $U_{q}(\hat{\mathbf{g}})$ has since played extremely important role in the theory of affine quantum algebras.

However from the structure point of view, Drinfeld realization is not complete in the sense that in this realization only the current operators for the simple roots are given. Also recently, it has gradually become clear that there is a possibility to develop a theory of Drinfeld realization as a theory of quantized algebras of currents completely parallel to the theory of quantum groups. This is the problem we want to address in this paper.

The receipt of contructing the new current operator follows from a simple idea in the theory of the affine Lie algebras, which is to use analytic properties of the matrix coefficients of current operators. Let $\alpha, \beta$ and $\alpha+\beta$ be positive roots of $\mathbf{g}$. In the affine Lie algebra $\hat{\mathbf{g}}$, we have

$$
\left[X_{\alpha}(z), X_{\beta}(w)\right]=\delta(z / w) X_{\alpha+\beta}(z)
$$

\footnotetext{
${ }^{1}$ E-mail: ding@kurims.kyoto-u.ac.jp

${ }^{2}$ E-mail: khoroshkin@vitep5.itep.ru
} 
where $z$ and $w$ are formal variables and $\delta(z / w)=\Sigma_{n \in \mathbf{Z}}(z / w)^{n}$. Let $V_{\lambda}$ be an highest weight representation of $\hat{\mathbf{g}}, V_{\lambda}^{*}$ its dual, $v \in V_{\lambda}$ and $v^{*} \in V_{\lambda}^{*}$. We know that $\left\langle v^{*}, X_{\alpha}(z) X_{\beta}(w)>\right.$ as Laurent series converges if we substitute $z$ and $w$ with numbers in the region $|w|<<|z|$. From such a point of view, we can see that $X_{\alpha+\beta}(z)$ actually is an operator sitting on the pole of $X_{\alpha}(z) X_{\beta}(w)$, which is $(z-w)=0$. Thus analytically we can rewrite it as

$$
\int_{z \text { around } w} X_{\alpha}(z) X_{\beta}(w) d z / z=X_{\alpha+\beta}(w),
$$

which is a well-known formula in field theory. In the quantum case, we use a modification of (0.1). The formula are of the following type:

$$
Y_{\alpha+\beta}(w)=\int_{z \text { around } w q^{ \pm 1}} Y_{\alpha}(z) Y_{\beta}(w) d z / z
$$

where $Y_{\alpha}(z)$ and $Y_{\beta}(w)$ are current operators for the simple roots from Drinfeld realization of $U_{q}(\hat{\mathbf{g}})$. The form of the commutation relations for the current operators of Drinfeld realization and the properties of highest weight representations give us the possibility to define precisely the analytic continuation of the products of current operators. This allows us to define R.H.S. of (0.2) as an element of algebra. Applying this method further, we derive the whole set of current operator corresponding to all the roots of $\mathbf{g}$ for $U_{q}(\hat{\mathbf{g}})$.

As we know in the classical case, the Weyl group is an important and inseperable structure in the theory of simple Lie algebra, once the theory of root system is established. Going further in the develpment of the theory of quantized current algebras, we define an action of the braid group on $U_{q}(\widehat{\mathbf{g}})$ in terms of currents. This action uses, in particular, the composed root currents and does not coincide with Lusztig automorphisms [[] of $U_{q}(\widehat{\mathbf{g}})$. The automorphisms form a semidirect product of the braid group with the weight lattice. Beyond that we manage to derive a new Hopf algebra by combining both braid group automorphisms and $U_{q}(\hat{\mathbf{g}})$.

Current realization of quantum affine algebra is naturally supplied with its own quasitriangular Hopf algebra structure (also given by Drinfeld [Dr3]), which is different from that for quantum groups. This Hopf algebra structure can be derived as a limit of twists of the Hopf algebra structure for affine quantum groups given by Drinfeld and Jimbo [KT3]. The results of [KT3 also give the formula for the corresponding universal R-matrix, which, however, is not given in the form of current operators and is not effictive for algebras of rank higher than two. An attempt to derive the universal R-matrix for the current realization for $U_{q}\left(\hat{\mathbf{s}}_{2}\right)$ was taken in [ER]. However, their naive formula is not correct. We derive a formula the universal R-matrix using a type of noncommutative functional generalization of exponential functions, where the choice of integration contours plays a fundamental role. The formula is given first for the case of $U_{q}\left(\hat{\mathbf{s}}_{2}\right)$ (see (3.20) ) and then is extended to general case by the Weyl group technique. Simulteniously we get a presentation of the universal $R$-matrix in a form of infinite product different from [KT3 for generic g. For comparison, we give in Appendix the computation of quadratic terms of universal $R$-matrix for $U_{q}\left(\hat{\mathbf{s l}}_{2}\right)$ in both approaches.

The integral form of the universal $R$-matrix is given in a series of integrals which, from one side, look as the complex-analytical counterpart of ordered exponentials over the real variable; the order of the arguments is replaced by the precise choise of the poles inside the integration region. On the other hand, the properties of the integrals are direct generalizations of the properties of $q$-exponential functions [FV], [KT1, [V]. We observe them in a separate section. One can speculate further for current generalizations of the quantum plane.

Our considerations are of quite general nature and can be applied as well to the Yangians and to the face type elliptic algebras. In section 5 we presents such results for the double of 
the Yangians. It is also clear how to extend them for non-simple laced case. Note also that due to the results in [BLZ], we can suppose that there should exist fumdamental presentations of the universal $R$-matrices in an integral form of ordered exponentials and the formulas which we present here could be viewed as an approximation to these presentations.

\section{Composed root currents.}

Let $\mathbf{g}$ be a simple Lie algebra of simple laced type and $U_{q}^{(D)}(\widehat{\mathbf{g}})$ be the corresponding quantum affine algebra. By $U_{q}^{(D)}(\widehat{\mathbf{g}})$ we mean that first, we define the algebra by the "new realization" of $U_{q}(\widehat{\mathbf{g}})$ in generating functions by V. Drinfeld [Dr2], second, this algebra has the Hopf algebra structure, naturally attached to this realization [Dr3] and third, we restrict this algebra to the category of highest weight representations.

The generators of the algebra $U_{q}^{(D)}(\widehat{\mathbf{g}})$ are given by generating functions $e_{\alpha_{i}}(u), f_{\alpha_{i}}(u)$ and $K_{\alpha_{i}}^{ \pm}(u)$ :

$$
e_{\alpha_{i}}(z)=\sum_{k \in \mathbf{Z}} e_{\alpha_{i}, k} z^{-k}, \quad f_{\alpha_{i}}(z)=\sum_{k \in \mathbf{Z}} f_{\alpha_{i}, k} z^{-k}
$$

and

$$
K_{\alpha_{i}}^{ \pm}(z)=k_{\alpha_{i}}^{ \pm 1} \exp \left( \pm\left(q-q^{-1}\right) \sum_{n>0} a_{i, \pm n} z^{\mp n}\right) .
$$

where $\alpha_{i}, i=1, \ldots, r$ are all simple roots of $\mathbf{g}$.

They satisfy the following defining relations $(\alpha, \beta$ are simple roots of $\mathbf{g})$ :

$$
\begin{aligned}
& \left(z-q^{(\alpha, \beta)} w\right) e_{\alpha}(z) e_{\beta}(w)=e_{\beta}(w) e_{\alpha}(z)\left(q^{(\alpha, \beta)} z-w\right), \\
& \left(z-q^{-(\alpha, \beta)} w\right) f_{\alpha}(z) f_{\beta}(w)=f_{\beta}(w) f_{\alpha}(z)\left(q^{-(\alpha, \beta)} z-w\right), \\
& \frac{\left(q^{c / 2} z-q^{(\alpha, \beta)} w\right)}{\left(q^{(\alpha, \beta)+c / 2} z-w\right)} K_{\alpha}^{+}(z) e_{\beta}(w)=e_{\beta}(w) K_{\alpha}^{+}(z), \\
& K_{\alpha}^{-}(z) e_{\beta}(w)=\frac{\left(q^{(\alpha, \beta)-c / 2} z-w\right)}{\left(q^{-c / 2} z-q^{(\alpha, \beta)} w\right)} e_{\beta}(w) K_{\alpha}^{-}(z), \\
& \frac{\left(q^{-c / 2} z-q^{-(\alpha, \beta)} w\right)}{\left(q^{-(\alpha, \beta)-c / 2} z-w\right)} K_{\alpha}^{+}(z) f_{\beta}(w)=f_{\beta}(w) K_{\alpha}^{+}(z), \\
& K_{\alpha}^{-}(z) f_{\beta}(w)=\frac{\left(q^{-(\alpha, \beta)+c / 2} z-w\right)}{\left(q^{c / 2} z-q^{-(\alpha, \beta)} w\right)} f_{\beta}(w) K_{\alpha}^{-}(z), \\
& \frac{\left(z-q^{(\alpha, \beta)-c} w\right)\left(z-q^{-(\alpha, \beta)+c} w\right)}{\left(q^{(\alpha, \beta)+c} z-w\right)\left(q^{-(\alpha, \beta)-c} z-w\right)} K_{\alpha}^{+}(z) K_{\beta}^{-}(w)=K_{\beta}^{-}(w) K_{\alpha}^{+}(z) \\
& K_{\alpha}^{ \pm}(z) K_{\beta}^{ \pm}(w)=K_{\beta}^{ \pm}(w) K_{\alpha}^{ \pm}(z) \\
& {\left[e_{\alpha}(z), f_{\beta}(w)\right]=\frac{\delta_{\alpha, \beta}}{q-q^{-1}}\left(\delta\left(z / q^{c} w\right) K_{\alpha}^{+}\left(z q^{-c / 2}\right)-\delta\left(z q^{c} / w\right) K_{\alpha}^{-}\left(w q^{-c / 2}\right)\right)}
\end{aligned}
$$

and the Serre relations:

$$
\begin{aligned}
& e_{\alpha}\left(w_{1}\right) e_{\alpha}\left(w_{2}\right) e_{\beta}(z)-[2]_{q} e_{\alpha}\left(w_{1}\right) e_{\beta}(z) e_{\alpha}\left(w_{2}\right)+e_{\beta}(z) e_{\alpha}\left(w_{1}\right) e_{\alpha}\left(w_{2}\right)+w_{1} \leftrightarrow w_{2}=0 \\
& f_{\alpha}\left(w_{1}\right) f_{\alpha}\left(w_{2}\right) f_{\beta}(z)-[2]_{q} f_{\alpha}\left(w_{1}\right) f_{\beta}(z) f_{\alpha}\left(w_{2}\right)+f_{\beta}(z) f_{\alpha}\left(w_{1}\right) f_{\alpha}\left(w_{2}\right)+w_{1} \leftrightarrow w_{2}=0
\end{aligned}
$$


for any $\alpha, \beta,(\alpha, \beta)=-1$. Here $\delta(z)=\sum_{k \in \mathbf{Z}} z^{k}$.

All the relations are the equations on formal power series, but for the relations (1.3)-(1.10) and (1.12), (1.13), both sides of them are analytical functions with singularities at $z, w=0, \infty$ when they act on highest weight modules, while the relation (1.11) is given in the sense of analytical continuation. In general we follow the rule to understand the equalities without taking analytical continuations if the regions of Laurent expansions are not specified and there are no $\delta$ - functions terms.

In a highest weight representation, the operator valued functions $e_{\alpha}(z) e_{\beta}(w)$ are analytical in a region $|z|>\left|q^{(\alpha, \beta)} w\right|$ with the products $e_{\alpha, n} e_{\beta, m}$ being their Laurent coefficients in this region, and the relations (1.3) allow us to define the analytical continuation of these functions into a region $|z|<\left|q^{(\alpha, \beta)} w\right|$ as Laurent series

$$
\frac{q^{(\alpha, \beta)} z-w}{z-q^{(\alpha, \beta)} w} e_{\beta}(w) e_{\alpha}(z)
$$

The formula (1.14) can be considered as the definition of analytical continuations for the products of currents in algebra $U_{q}^{(D)}(\widehat{\mathbf{g}})$. On the other hand, the restriction to the category of highest weight representations dictates, that we can consider the equalities in terms of normal ordered series of generators of the algebra like

$$
\sum_{k_{1} \leq k_{2} \leq \cdots \leq k_{m}} C_{\alpha_{i}, k_{i}} e_{\alpha_{1}, k_{1}} e_{\alpha_{2}, k_{2}} \cdots e_{\alpha_{m}, k_{m}}
$$

The precise definition of the topological structure of the algebra $U_{q}^{(D)}(\widehat{\mathrm{g}})$ is given in [KT3], [DK].

This is the ideology we use throughout the paper.

For any two simple roots $\alpha$ and $\beta$ let $e_{\alpha+\beta}(z)$ and $f_{\alpha+\beta}(z)$ be the currents

$$
e_{\alpha+\beta}(z)=: e_{\alpha}(q z) e_{\beta}\left(q^{2} z\right):, \quad f_{\alpha+\beta}(z)=: f_{\beta}\left(q^{2} z\right) f_{\alpha}(q z):,
$$

that is

$$
e_{\alpha+\beta}(z)=\frac{1}{2 \pi i} \oint_{w \text { around } q z} e_{\alpha}(w) e_{\beta}\left(q^{2} z\right) \frac{d w}{w}, \quad f_{\alpha+\beta}(z)=\frac{1}{2 \pi i} \oint_{w \text { around } q z} f_{\beta}\left(q^{2} z\right) f_{\alpha}(w) \frac{d w}{w},
$$

where the integrands are taken in a sense of analytical continuations. Note that $e_{\alpha+\beta}(z) \neq$ $e_{\beta+\alpha}(z)$. Performing the analytical continuation, we can rewrite the definition (1.15) as a difference of two contour integrals:

$$
\begin{aligned}
& e_{\alpha+\beta}(z)=\frac{1}{2 \pi i}\left(\oint_{C_{\infty}} e_{\alpha}(w) e_{\beta}\left(q^{2} z\right) \frac{d w}{w}-\oint_{C_{0}} \frac{q^{-1} w-q^{2} z}{w-q z} e_{\beta}\left(q^{2} z\right) e_{\alpha}(w) \frac{d w}{w}\right), \\
& f_{\alpha+\beta}(z)=\frac{1}{2 \pi i}\left(-\oint_{C_{0}} f_{\beta}\left(q^{2} z\right) f_{\alpha}(w) \frac{d w}{w}+\oint_{C_{\infty}} \frac{q^{-1} w-q^{2} z}{w-q z} f_{\alpha}(w) f_{\beta}\left(q^{2} z\right) \frac{d w}{w}\right),
\end{aligned}
$$

where $C_{0}$ is a contour around zero such that the point $w=q z$ is outside the contour while $C_{\infty}$ is a contour close to infinity, which includes zero and the point $w=q z$. In other words,

$$
\begin{array}{cc}
e_{\alpha}(z) e_{\beta}(w)-\frac{q^{-1} z-w}{z-q^{-1} w} e_{\beta}(w) e_{\alpha}(z)=\delta(z q / w) e_{\alpha+\beta}\left(q^{-1} z\right), & |z|<\left|q^{-1} w\right| \\
f_{\beta}(z) f_{\alpha}(w)-\frac{q z-w}{z-q w} f_{\alpha}(w) f_{\beta}(z)=\delta(z / q w) f_{\alpha+\beta}\left(q^{-1} w\right), & |z|<|q w|
\end{array}
$$


In terms of Fourier modes $e_{\gamma, n}=\frac{1}{2 \pi i} \oint e_{\gamma}(w) w^{n} \frac{d w}{w}$, the definitions (1.16) and (1.17) can be rewritten as

$$
\begin{gathered}
e_{\alpha+\beta, n}=q^{-2 n-p}\left(e_{\alpha, p} e_{\beta, n-p}-q e_{\beta, n-p} e_{\alpha, p}-\left(q-q^{-1}\right) \sum_{k \geq 1} q^{k} e_{\beta, n-p-k} e_{\alpha, p+k}\right) \\
f_{\alpha+\beta, n}=q^{-2 n-p}\left(-f_{\beta, n-p} f_{\alpha, p}+q^{-1} f_{\alpha, p} f_{\beta, n-p}-\left(q-q^{-1}\right) \sum_{k \geq 1} q^{-k} f_{\alpha, p-k} f_{\beta, n-p+k}\right)
\end{gathered}
$$

for any integer $p$.

Proposition 1 The following relations are equivalent to the Serre relations:

$$
\begin{gathered}
e_{\alpha}(z) e_{\alpha+\beta}(w)=\frac{q z-w}{z-q w} e_{\alpha+\beta}(w) e_{\alpha}(z), \quad|z|<|q w|, \\
e_{\alpha+\beta}(z) e_{\beta}(w)=\frac{q^{2} z-q^{-2} w}{q z-q^{-1} w} e_{\beta}(w) e_{\alpha+\beta}(z), \quad|z|<\left|q^{-2} w\right|, \\
f_{\beta}(z) f_{\alpha+\beta}(w)=\frac{q^{-2} z-q^{2} w}{q^{-1} z-q w} f_{\alpha+\beta}(w) f_{\alpha}(z), \quad|z|<\left|q^{2} w\right|, \\
f_{\alpha+\beta}(z) f_{\alpha}(w)=\frac{q^{-1} z-w}{z-q^{-1} w} f_{\alpha}(w) f_{\alpha+\beta}(z), \quad|z|<\left|q^{-1} w\right|,
\end{gathered}
$$

The equalities (1.22)-(1.25) are the equalities of Laurent series. In analytical language they are equalities of the functions, analytical in $\left(\mathbf{C}^{*}\right)^{2}$ without restrictions to a region.

Proof. Let us examine the relation (1.24). Due to the Serre relations and (1.4), (1.19), we have

$$
\begin{gathered}
f_{\alpha}\left(w_{1}\right) f_{\alpha}\left(w_{2}\right) f_{\beta}(z)-\left(q+q^{-1}\right) f_{\alpha}\left(w_{1}\right) f_{\beta}(z) f_{\alpha}\left(w_{2}\right)+f_{\beta}(z) f_{\alpha}\left(w_{1}\right) f_{\alpha}\left(w_{2}\right)= \\
\frac{\left(q-q^{-1}\right) z\left(w_{1}-q^{-2} w_{2}\right)}{\left(w_{2}-q^{-1} z\right)\left(w_{1}-q^{-1} z\right)} f_{\alpha}\left(w_{1}\right) f_{\alpha}\left(w_{2}\right) f_{\beta}(z)+f_{\alpha+\beta}\left(q^{-1} w_{1}\right) f_{\alpha}\left(w_{2}\right) \delta\left(z / q w_{1}\right)+ \\
\frac{q z-w_{1}}{z-q w_{1}} f_{\alpha}\left(w_{1}\right) f_{\alpha+\beta}\left(q^{-1} w_{2}\right) \delta\left(z / q w_{2}\right)-\left(q-q^{-1}\right) f_{\alpha}\left(w_{1}\right) f_{\alpha+\beta}\left(q^{-1} w_{2}\right) \delta\left(z / q w_{2}\right)= \\
\frac{\left(q-q^{-1}\right) z\left(w_{1}-q^{-2} w_{2}\right)}{\left(w_{2}-q^{-1} z\right)\left(w_{1}-q^{-1} z\right)} f_{\alpha}\left(w_{1}\right) f_{\alpha}\left(w_{2}\right) f_{\beta}(z)+ \\
f_{\alpha+\beta}\left(q^{-1} w_{1}\right) f_{\alpha}\left(w_{2}\right) \delta\left(z / q w_{1}\right)-\frac{q^{-1} z-q^{2} w_{1}}{z-q w_{1}} f_{\alpha}\left(w_{1}\right) f_{\alpha+\beta}\left(q^{-1} w_{2}\right) \delta\left(z / q w_{2}\right) .
\end{gathered}
$$

Then we do the same with arguments $w_{1}$ and $w_{2}$ interchanged and summ up. The terms without delta functions dissapear while the coefficients before delta functions give the same equation

$$
f_{\alpha+\beta}\left(q^{-1} w_{1}\right) f_{\alpha}\left(w_{2}\right)=\frac{w_{1}-q^{2} w_{2}}{q w_{1}-q w_{2}} f_{\alpha}\left(w_{2}\right) f_{\alpha+\beta}\left(q^{-1} w_{1}\right),
$$

which is equivalent to (1.24). It is easy to see that the arguments can be reversed in order to deduce from (1.24) one of the Serre relations.

In particular 1.22 - 1.25) imply an analog of PBW theorem for the Fourier components of the currents for rank three algebra $U_{q}^{(D)}\left(\widehat{\mathbf{s l}}_{\mathbf{3}}\right)$. 
In the following we need also another pair of composed root currents:

$$
\check{e}_{\alpha+\beta}(z)=: e_{\beta}\left(q^{-2} z\right) e_{\alpha}\left(q^{-1} z\right):, \quad \check{f}_{\alpha+\beta}(z)=: f_{\alpha}\left(q^{-1} z\right) f_{\beta}\left(q^{-2} z\right):,
$$

that is,

$$
\begin{gathered}
\check{e}_{\alpha+\beta}(z)=\frac{1}{2 \pi i}\left(-\oint_{C^{\prime}{ }_{0}} e_{\beta}\left(q^{-2} z\right) e_{\alpha}(w) \frac{d w}{w}+\oint_{C^{\prime} \infty} \frac{q w-q^{-2} z}{w-q^{-1} z} e_{\alpha}(w) e_{\beta}\left(q^{-2} z\right) \frac{d w}{w}\right), \\
\check{f}_{\alpha+\beta}(z)=\frac{1}{2 \pi i}\left(\oint_{C^{\prime} \infty} f_{\alpha}(w) f_{\beta}\left(q^{-2} z\right) \frac{d w}{w}-\oint_{C^{\prime}{ }_{0}} \frac{q w-q^{-2} z}{w-q^{-1} z} f_{\beta}\left(q^{-2} z\right) f_{\alpha}(w) \frac{d w}{w}\right),
\end{gathered}
$$

where $C^{\prime}{ }_{0}$ is a contour around zero such that the point $w=q^{-1} z$ is outside the contour while $C^{\prime}{ }_{\infty}$ is a contour close to infinity, which includes zero and the point $w=q^{-1} z$.

In the following (see section 4 ), we define the composed root vectors for all roots of $\mathbf{g}$. As usual, the definition depends on the choise of reduced decomposition for the longest element $w_{0}$ of the Weyl group of $\mathbf{g}$.

\section{The automorphisms}

In this section we define a new braid group action on $U_{q}^{(D)}(\widehat{\mathbf{g}})$ using the current operators defined above.

For any simple root $\alpha$ let $T_{\alpha}$ be the following linear map:

$$
\begin{gathered}
T_{\alpha} e_{\alpha}(z)=f_{\alpha}\left(q^{-c} z\right) K_{\alpha}^{+}\left(q^{-c / 2} z\right)^{-1}, \\
T_{\alpha} f_{\alpha}(z)=K_{\alpha}^{-}\left(q^{-c / 2} z\right)^{-1} e_{\alpha}\left(q^{-c} z\right), \\
T_{\alpha} K_{\alpha}^{ \pm}(z)=K_{\alpha}^{ \pm}(z)^{-1},
\end{gathered}
$$

and for the simple $\beta,(\alpha, \beta)=-1$

$$
\begin{gathered}
T_{\alpha} K_{\beta}^{ \pm}(z)=K_{\alpha}^{ \pm}(q z) K_{\beta}^{ \pm}\left(q^{2} z\right), \\
T_{\alpha} e_{\beta}(z)=e_{\alpha+\beta}(z), \quad T_{\alpha} f_{\beta}(z)=f_{\alpha+\beta}(z) .
\end{gathered}
$$

The rest of Drinfeld current operators for simple roots is stable. We introduce the maps

$$
\begin{gathered}
T_{\alpha}^{-1} e_{\alpha}(z)=K_{\alpha}^{-}\left(q^{c / 2} z\right)^{-1} f_{\alpha}\left(q^{c} z\right) \\
T_{\alpha}^{-1} f_{\alpha}(z)=e_{\alpha}\left(q^{c} z\right) K_{\alpha}^{+}\left(q^{c / 2} z\right)^{-1} \\
T_{\alpha}^{-1} K_{\alpha}^{ \pm}(z)=K_{\alpha}^{ \pm}(z)^{-1}
\end{gathered}
$$

and for the simple $\beta,(\alpha, \beta)=-1$

$$
\begin{gathered}
T_{\alpha}^{-1} K_{\beta}^{ \pm}(z)=K_{\alpha}^{ \pm}\left(q^{-1} z\right) K_{\beta}^{ \pm}\left(q^{-2} z\right), \\
T_{\alpha}^{-1} e_{\beta}(z)=\check{e}_{\alpha+\beta}(z), \quad T_{\alpha}^{-1} f_{\beta}(z)=\check{f}_{\alpha+\beta}(z) .
\end{gathered}
$$

We also define the following automorphisms of affine shift. For any simple root $\alpha$ we put

$$
P_{\omega_{\alpha}} e_{\alpha}(z)=z e_{\alpha}(z), \quad P_{\omega_{\alpha}} f_{\alpha}(z)=z^{-1} f_{\alpha}(z),
$$




$$
P_{\omega_{\alpha}} K_{\alpha}^{ \pm}(z)=q^{\mp c} K_{\alpha}^{ \pm}(z)
$$

The rest of of Drinfeld current operators for simple roots is stable. Here $\omega_{\alpha}$ denotes $\alpha$-th fundamental weight: $\left(\omega_{\alpha}, \beta\right)=\delta_{\alpha, \beta}$.

The maps $P_{\omega_{\alpha}}$ and $P_{\omega_{\beta}}$ commute for different roots, so for any element $\lambda$ of a weight lattice $Q$ of Lie algebra $\mathbf{g}$ there is well defined automorphism $P_{\lambda}$.

Proposition 2 The maps $T_{\alpha}^{ \pm 1}$ and $P_{\lambda}$ can be extended to the automorphisms of the algebra $U_{q}^{(D)}(\widehat{\mathbf{g}})$. They form a semidirect product of a braid group with a lattice $Q$, that is, $T_{\alpha}$ satisfy the braid group relations

$$
\begin{gathered}
T_{\alpha} T_{\beta} T_{\alpha}=T_{\beta} T_{\alpha} T_{\beta}, \quad(\alpha, \beta)=-1, \\
T_{\alpha} T_{\beta}=T_{\beta} T_{\alpha}, \quad(\alpha, \beta)=0, \\
T_{\alpha} T_{\alpha}^{-1}=T_{\alpha}^{-1} T_{\alpha}=1
\end{gathered}
$$

and reflect the shift automorphisms $P_{\lambda}$ :

$$
T_{\alpha} P_{\lambda}=P_{s_{\alpha}(\lambda)} T_{\alpha}
$$

Here $s_{\alpha}$ is a reflection in $\mathbf{h}^{*}$ with respect to a root $\alpha: s_{\alpha}(\lambda)=\lambda-2 \frac{(\alpha, \lambda)}{(\alpha, \alpha)} \alpha$.

The proof of the automorphisms properties and of the braid group relations (2.13), (2.14) and of (2.16) consists of direct verification for rank two and rank three algebras.

Let us make several remarks on the definition of automorphisms. One can see that the action of braid group generators $T_{\alpha}^{ \pm 1}$ is quite different from that of Lusztig automorphisms [[]]. To the contrary, the affine shifts are actually present in usual quantum Weyl group of $U_{q}(\widehat{\mathrm{g}})$, extended by the automorphisms of a Dynkin diagramm $\mathrm{Be}$. There are also two subtle things in the formulas (2.1)-(2.5) and in the commutation relations of composed root currents, which do not appear in finite- dimensional case. The first one is the shifts of the arguments in commutation relations (1.23) and (1.25) and in the definition of the action of braid group generators (2.2), (2.7) by $q$. We have no satisfactory explanation of this shift.

The second one is the inverse order in the relations (1.22) and (1.24), (1.23) and (1.25). It is not as for usual Lusztig automorphisms which mean that the order of negative currents in PBW is opposite to the one for the positives.

We denote by $W_{q}(\widehat{\mathbf{g}})$ the corresponding group of automorphisms and by $W_{q}(\widehat{\mathbf{g}}) \times U_{q}^{(D)}(\widehat{\mathbf{g}})$ the associated semidirect product of an algebra $\mathbf{C} \cdot W_{q}(\widehat{\mathbf{g}})$ and of $U_{q}^{(D)}(\widehat{\mathbf{g}})$. We define it by the relations

$$
T_{\alpha} \cdot x \cdot T_{\alpha}^{-1}=T_{\alpha}(x), \quad P_{\lambda} \cdot x \cdot P_{\lambda}^{-1}=P_{\lambda}(x)
$$

where $x \in U_{q}^{(D)}(\widehat{\mathbf{g}})$.

\section{Universal $R$-matrices for $U_{q}^{(D)}\left(\widehat{\mathbf{s l}}_{2}\right)$}

There are two standard divisions of $U_{q}^{(D)}\left(\widehat{\mathbf{s l}}_{\mathbf{2}}\right)$ into a sum of opposite Borel subalgebras. The two different comultiplication rules correspond to them. Let us first consider the possibility, which we denote further by (I), where the following comultiplication rules take place (we write them for all $U_{q}^{(D)}(\widehat{\mathbf{g}})$ and omit an index of a simple root for $\left.U_{q}^{(D)}\left(\widehat{\mathbf{s l}}_{\mathbf{2}}\right)\right)$

$$
\Delta_{(I)} e_{\alpha}(z)=e_{\alpha}(z) \otimes 1+K_{\alpha}^{-}\left(z q^{c_{1} / 2}\right) \otimes e_{\alpha}\left(z q^{c_{1}}\right)
$$




$$
\begin{gathered}
\Delta_{(I)} f_{\alpha}(z)=1 \otimes f_{\alpha}(z)+f_{\alpha}\left(z q^{c_{2}}\right) \otimes K_{\alpha}^{+}\left(z q^{c_{2} / 2}\right), \\
\Delta_{(I)} K_{\alpha}^{+}(z)=K_{\alpha}^{+}\left(z q^{c_{2} / 2}\right) \otimes K_{\alpha}^{+}\left(z q^{-c_{1} / 2}\right), \\
\Delta_{(I)} K_{\alpha}^{-}(z)=K_{\alpha}^{-}\left(z q^{-c_{2} / 2}\right) \otimes K_{\alpha}^{-}\left(z q^{c_{1} / 2}\right),
\end{gathered}
$$

Let us treate the Hopf algebra $U_{q}^{(D)}\left(\widehat{\mathbf{s l}}_{\mathbf{2}}\right)$ as a double of subalgebra, generated by $f(z)$ and $K^{+}(z)$.

The results by [KT3], see also [KT2] imply that the universal $R$-matrix for the first case with a restriction $|q|<1$ has a form (in order to adapt the notations [KT3] to (1.3)-(1.13) one should change $q$ to $q^{-1}$ everywhere in the formulas presented in [KT3]:

$$
\mathcal{R}_{(I)}=\mathcal{K} \cdot \overline{\mathcal{R}}
$$

where

$$
\mathcal{K}=q^{-\frac{h \otimes h}{2}} q^{\frac{-c \otimes d-d \otimes c}{2}} \exp \left(-\left(q-q^{-1}\right) \sum_{n>0} \frac{n}{[2 n]_{q}} a_{n} \otimes a_{-n}\right) q^{\frac{-c \otimes d-d \otimes c}{2}}, \quad d=-z \frac{d}{d z}
$$

and

$$
\overline{\mathcal{R}}=\prod_{n \in \mathbf{Z}} \exp _{q^{2}}\left(-\left(q-q^{-1}\right) f_{-n} \otimes e_{n}\right)
$$

Here

$$
\exp _{p}(x)=1+x+\frac{x^{2}}{1+p}+\frac{x^{3}}{(1+p)\left(1+p+p^{2}\right)}+\ldots+\frac{x^{n}}{(n)_{p} !}+\ldots
$$

The tensor of the Hopf pairing in $U_{q}^{(D)}(\widehat{\mathrm{g}})$ when it is considered as a double of of subalgebra, generated by $e(z)$ and $K^{-}(z)$ is, by definition, $\left(\mathcal{R}_{(I)}{ }^{21}\right)^{-1}$. It has a form

$$
\left(\mathcal{R}_{(I)}^{21}\right)^{-1}=\overline{\mathcal{R}}^{\prime} \cdot \mathcal{K}^{\prime}
$$

where

$$
\mathcal{K}^{\prime}=\left(\mathcal{K}^{21}\right)^{-1}=q^{\frac{h \otimes h}{2}} q^{\frac{c \otimes d+d \otimes c}{2}} \exp \left(\left(q-q^{-1}\right) \sum_{n>0} \frac{n}{[2 n]_{q}} a_{-n} \otimes a_{n}\right) q^{\frac{c \otimes d+d \otimes c}{2}}
$$

and

$$
\overline{\mathcal{R}}^{\prime}=\left(\overline{\mathcal{R}}^{21}\right)^{-1}=\prod_{n \in \mathbf{Z}} \exp _{q^{-2}}\left(\left(q-q^{-1}\right) e_{-n} \otimes f_{n}\right) .
$$

Below we derive an integral form of the expressions (3.7) and (3.10), using the current operators $e(z)$ and $f(z)$. In order to do this we study the pairing of two Hopf dual Borel subalgebras in $U_{q}^{(D)}\left(\widehat{\mathbf{s l}}_{\mathbf{2}}\right)$.

We will follow the convention that in the pairing of Hopf algebras $A$ and $A^{0}$, where $A^{0}$ is Hopf dual with opposite comultiplication, the elements of $A$ stand in the first place. In other words, we use the rules

$$
<a b, x>=<a \otimes b, \Delta^{\prime}(x)>, \quad<a, x y>=<\Delta(a), x \otimes y>.
$$

Let us remind, that the commutation relations for the two subralgeras of a quantum double are given, due to Drinfeld, by the folowing rule:

$$
a \cdot b=<a^{(1)}, b^{(1)}><S^{-1}\left(a^{(3)}\right), b^{(3)}>b^{(2)} \cdot a^{(2)}
$$


where $a \in A, b \in A^{0}, \Delta^{2}(x)=(\Delta \otimes I d) \Delta(x)=x^{(1)} \otimes x^{(2)} \otimes x^{(3)}, S$ is antipode in $A$. Then, applying (3.12) to check of the relation (1.11) we have:

$$
<f(z), e(w)>=-\frac{\delta(z / w)}{q-q^{-1}}
$$

Then the relation (3.11), applied to commutation relation (1.5)- (1.8), give the pairing for $K^{ \pm}(u)$ :

$$
<K^{+}(z), K^{-}(w)>=\frac{q^{2} z-w}{z-q^{2} w}, \quad|w|<\left|q^{-2} z\right| .
$$

which means that

$$
<k_{\alpha}, k_{\alpha}>=q^{-2}, \quad<h, h>=-\frac{1}{\log q}, \quad<a_{n}, a_{-n}>=-\frac{[2 n]_{q}}{n\left(q-q^{-1}\right)}
$$

where $h=\log _{q} k_{\alpha}$. Moreover, the rule (3.11) gives the following triangular decomposition of the pairing:

$$
<K^{+}\left(z_{1}\right) f\left(z_{2}\right), e(w)>=<f\left(z_{2}\right), e(w)>, \quad<f(z), K^{-}\left(w_{1}\right) e\left(w_{2}\right)>=<f(z), e\left(w_{2}\right)>.
$$

This produces factorization (3.5) of the corresponding universal $R$-matrix. The further study of the pairing of the products of fields $K^{+}\left(z_{i}\right)$ and of $K^{-}\left(w_{j}\right)$ gives the factor $\mathcal{K}$. We omit these calculations.

By induction, one can deduce the following description for the pairing of the products of fields $f\left(z_{i}\right)$ and $e\left(w_{j}\right)$ :

$$
\begin{gathered}
<f\left(z_{1}\right) f\left(z_{2}\right) \cdots f\left(z_{n}\right), e\left(w_{1}\right) e\left(w_{2}\right) \cdots e\left(w_{n}\right)>= \\
\frac{1}{\left(q^{-1}-q\right)^{n}} \sum_{\sigma \in S_{n}} \prod_{i=1}^{n} \delta\left(\frac{z_{i}}{\sigma\left(w_{j}\right)}\right) \prod_{\substack{i<j, \sigma(i)>\sigma(j)}} g\left(\frac{z_{i}}{z_{j}}\right),
\end{gathered}
$$

where the function

$$
g(z)=\frac{q^{2}-z}{1-q^{2} z}, \quad|z|<q^{-2}
$$

is expanded in the mentioned region of analyticity $|z|<q^{-2}$. The relation (3.17) is an equality of formal power series.

The tensor $\overline{\mathcal{R}}$ diagonilizes this pairing. It can be written in the following ordered exponential form:

\section{Proposition 3}

$$
\overline{\mathcal{R}}=\mathcal{P} \overrightarrow{\exp }_{q^{2}}\left(\frac{\left(q^{-1}-q\right)}{2 \pi i} \oint f(z) \otimes e(z) \frac{d z}{z}\right),
$$

where

$$
\begin{gathered}
\mathcal{P} \overrightarrow{\exp }_{q^{2}}\left(\frac{\left(q^{-1}-q\right)}{2 \pi i} \oint f(z) \otimes e(z) \frac{d z}{z}\right)=1+ \\
\sum_{n>0} \frac{\left(q^{-1}-q\right)^{n}}{n !(2 \pi i)^{n}} \oint_{C_{n}} \frac{d z_{n}}{z_{n}} \oint_{C_{n-1}} \frac{d z_{n-1}}{z_{n-1}} \cdots \oint_{C_{1}} \frac{d z_{1}}{z_{1}} f\left(z_{1}\right) f\left(z_{2}\right) \ldots f\left(z_{n}\right) \otimes \\
e\left(z_{1}\right) e\left(z_{2}\right) \ldots e\left(z_{n}\right),
\end{gathered}
$$

and the contours $C_{k}$ enclose an origin and are in a region where the points $z_{i}=q^{2} z_{j}$ are outside the contours $C_{i}$ and the points $z_{i}=q^{-2} z_{j}$ are inside the contours $C_{i}$ 
One can choose the contours as follows. First, all the contours contain an origin. Then, the contour $C_{1}$ contains the points $q^{-2} z_{j}, j>1$ and the points $q^{2} z_{j}, j>1$ are outside. The contour $C_{2}$ contains the points $q^{-2} z_{j}, j>2$ and the points $q^{2} z_{j}, j>2$ are outside and so on.

Note that in order to get an expression for $\overline{\mathcal{R}}$ in a component form, one should first analytically continuate the integrand of (3.20) into the region (suppose, for instance, $|q|>1$ )

$$
\left|q^{-2} z_{j}\right|<\left|z_{i}\right|<\left|q^{2} z_{j}\right|, \quad i \neq j
$$

and expand this continuation:

$$
\prod_{i<j} \frac{q^{-2}-z_{i} / z_{j}}{1-q^{-2} z_{i} / z_{j}} f\left(z_{1}\right) f\left(z_{2}\right) \ldots f\left(z_{n}\right) \otimes e\left(z_{n}\right) e\left(z_{n-1}\right) \ldots e\left(z_{1}\right)
$$

into Laurent series in the region (3.21). The result does not depend on a restriction on $|q|$.

The second remark is about difference of the pictures for $|q|<1$ and $|q|>1$. We see, that for $|q|>1$ the domain of integration has a natural form while for $|q|<1$ we have a sofisticated picture of the contours. On the other hand, the behaviour of the factors in the infinite products (3.6) and (3.7) is also different for different values of $|q|$. The evaluation of the universal $R$ matrix (3.5) in a tensor product of two-dimensional representations $V\left(z_{1}\right) \otimes V\left(z_{2}\right)(V$ has a basis $\left.v_{0}, v_{1}\right)$ gives a triangular $R$-matrix with $\delta$-function term outside the diagonal [KT3] for $|q|<1$ and is purely diagonal for $|q|>1$ This is because the scalar factor

$$
<v_{0}\left|\mathcal{R}^{(I)}\right| v_{0}>=\exp \left(-\sum_{n>0} \frac{\left(q^{n}-q^{-n}\right)^{2}}{q^{2 n}-q^{-2 n}} \frac{z^{n}}{n}\right)
$$

coming from the action of bozons $a_{n}$ on $V(z)$ [KT3], [DI]:

$$
a_{ \pm n} v_{0}=\frac{q^{n}-q^{-n}}{q-q^{-1}} \frac{z^{ \pm n}}{n} v_{0}, \quad n>0
$$

admits different decompositions into infinite products for $|q|<1$ and $|q|>1$.

Proof. The main step is the translation of formal series pairing (3.17) into analytical language. We can prove that the relation

$$
\begin{gathered}
<f\left(z_{1}\right) f\left(z_{2}\right) \cdots f\left(z_{n}\right), e\left(w_{1}\right) e\left(w_{2}\right) \cdots e\left(w_{n}\right)>= \\
\frac{1}{\left(q^{-1}-q\right)^{n}} \sum_{\sigma \in S_{n}} \prod_{i=1}^{n} \delta\left(\frac{z_{i}}{\sigma\left(w_{j}\right)}\right) \prod_{\substack{i<j, \sigma(i)>\sigma(j)}} \frac{q^{-2}-z_{j} / z_{i}}{1-q^{-2} z_{j} / z_{i}},
\end{gathered}
$$

is valid as an equality of analytical functions in a region $\left|z_{i}\right|>\left|q^{-2} z_{j}\right|,\left|w_{i}\right|<\left|q^{2} w_{j}\right|$, where $i<j$. In order to prove (3.24), we rewrite first the formal power series equality (3.17) in a form

$$
\begin{gathered}
<f\left(z_{1}\right) f\left(z_{2}\right) \cdots f\left(z_{n}\right), e\left(w_{n}\right) e\left(w_{n-1}\right) \cdots e\left(w_{1}\right)>= \\
\frac{1}{\left(q^{-1}-q\right)^{n}} \sum_{\sigma \in S_{n}} \prod_{i=1}^{n} \delta\left(\frac{z_{i}}{\sigma\left(w_{n+1-j}\right)}\right) \prod_{\substack{i<j, \sigma(i)>\sigma(j)}} g\left(\frac{z_{i}}{z_{j}}\right),
\end{gathered}
$$

and then multiply both sides of (3.25) by a formal power series

$$
\prod_{\substack{i<j, \sigma(i)>\sigma(j)}} \bar{g}\left(\frac{w_{i}}{w_{j}}\right)
$$


where the function

$$
\bar{g}(w)=\frac{q^{-2}-w}{1-q^{-2} w}, \quad|w|<q^{2}
$$

is considered in the region $|w|<q^{2}$. Then in the rhs we have a cancellation of the rational functions over the same arguments, since they are expanded all in one direction, and, finally,

$$
\begin{gathered}
<f\left(z_{1}\right) f\left(z_{2}\right) \cdots f\left(z_{n}\right), \quad \prod_{\substack{i<j, \sigma(i)>\sigma(j)}} \bar{g}\left(\frac{w_{i}}{w_{j}}\right) e\left(w_{n}\right) e\left(w_{n-1}\right) \cdots e\left(w_{1}\right)>= \\
\frac{1}{\left(q^{-1}-q\right)^{n}} \sum_{\sigma \in S_{n}} \prod_{i=1}^{n} \delta\left(\frac{z_{i}}{\sigma\left(w_{j}\right)}\right) \prod_{\substack{i<j, \sigma(i)>\sigma(j)}} \frac{q^{-2}-z_{j} / z_{i}}{1-q^{-2} z_{j} / z_{i}},
\end{gathered}
$$

which is equivalent to (3.24) since both sides converge in the region $\left|z_{i}\right|>\left|q^{-2} z_{j}\right|,\left|w_{i}\right|<\left|q^{2} w_{j}\right|$, where $i<j$.

The rest follows from technical calculations. We should prove the following identities:

$$
<\overline{\mathcal{R}}, e\left(w_{1}\right) e\left(w_{2}\right) \cdots e\left(w_{n}\right) \otimes 1>=e\left(w_{1}\right) e\left(w_{2}\right) \cdots e\left(w_{n}\right)
$$

and

$$
<1 \otimes f\left(w_{1}\right) f\left(w_{2}\right) \cdots f\left(w_{n}\right), \overline{\mathcal{R}}>=f\left(w_{1}\right) f\left(w_{2}\right) \cdots f\left(w_{n}\right) .
$$

Let us show, how it works on quadratic terms.

Taking, for instance, $e\left(w_{1}\right) e\left(w_{2}\right) \otimes 1$ in the region

$$
\left|w_{1}\right|<q^{2}\left|w_{2}\right|
$$

we have due to 3.24$)$

$$
\begin{gathered}
\frac{\left(q-q^{-1}\right)^{2}}{2(2 \pi i)^{2}}<\oint_{C_{2}} \frac{d z_{2}}{z_{2}} \oint_{C_{1}} \frac{d z_{1}}{z_{1}} f\left(z_{1}\right) f\left(z_{2}\right) \otimes e\left(z_{1}\right) e\left(z_{2}\right), e\left(w_{1}\right) e\left(w_{2}\right) \otimes 1>= \\
\frac{1}{2(2 \pi i)^{2}} \oint_{C_{2}} \frac{d z_{2}}{z_{2}} \oint_{C_{1}} \frac{d z_{1}}{z_{1}} \delta\left(z_{1} / w_{1}\right) \delta\left(z_{2} / w_{2}\right) e\left(z_{1}\right) e\left(z_{2}\right)+ \\
\frac{1}{2(2 \pi i)^{2}} \oint_{C_{2}} \frac{d z_{2}}{z_{2}} \oint_{C_{1}} \frac{d z_{1}}{z_{1}} \frac{q^{-2}-z_{1} / z_{2}}{1-q^{-2} z_{1} / z_{2}} \delta\left(z_{1} / w_{2}\right) \delta\left(z_{1} / w_{2}\right) e\left(z_{1}\right) e\left(z_{2}\right)= \\
\frac{1}{2} e\left(w_{1}\right) e\left(w_{2}\right)+\frac{q^{-2}-w_{2} / w_{1}}{2\left(1-q^{-2} w_{2} / w_{1}\right)} e\left(w_{2}\right) e\left(w_{1}\right)
\end{gathered}
$$

The second summand coincides with the first one due to the restriction (3.29) on the region of analyticity. This gives the first desired equality. For the second case, it is convenient to perform the pairing of the tensor with $1 \otimes f\left(w_{1}\right) f\left(w_{2}\right)$ in a region $\left|w_{1}\right|>\left|q^{-2} w_{2}\right|$

A naive version of (3.19) was suggested in ER]. Note that one of the subtle points in the pairing (3.17) leading to a delicate answer is a restriction on a region in (3.18) which makes the pairing noncommutative.

The calculations for the case, where we consider $U_{q}\left(\widehat{\mathbf{s l}}_{\mathbf{2}}\right)$ as a double of subalgebra generated by the fields $e(z)$ and $K^{-}(z)$ are similar. The pairing (3.17) modulo signs is given by the same formula, and the only (but very important) difference is that the coeffitients are in another regions of analyticity:

$$
<e\left(w_{1}\right) e\left(w_{2}\right) \cdots e\left(w_{n}\right), f\left(z_{1}\right) f\left(z_{2}\right) \cdots f\left(z_{n}\right)>=
$$




$$
\frac{1}{\left(q-q^{-1}\right)^{n}} \sum_{\sigma \in S_{n}} \prod_{i=1}^{n} \delta\left(\frac{z_{i}}{\sigma\left(w_{j}\right)}\right) \prod_{\substack{i<j, \sigma(i)>\sigma(j)}} g\left(\frac{z_{i}}{z_{j}}\right)
$$

where the function $g(z)=\frac{q^{2}-z}{1-q^{2} z}$ is considered in the region $|z|>q^{-2}$.

As a result, we have

\section{Proposition 4}

$$
\overline{\mathcal{R}}^{\prime}=\left(\overline{\mathcal{R}}^{21}\right)^{-1}=\mathcal{P} \overrightarrow{\exp }_{q^{-2}}\left(\frac{\left(q-q^{-1}\right)}{2 \pi i} \oint e(z) \otimes f(z) \frac{d z}{z}\right)
$$

where

$$
\begin{gathered}
\mathcal{P} \overline{\exp }_{q^{-2}}\left(\frac{\left(q-q^{-1}\right)}{2 \pi i} \oint e(z) \otimes f(z) \frac{d z}{z}\right)=1+ \\
\sum_{n>0} \frac{\left(q-q^{-1}\right)^{n}}{n !(2 \pi i)^{n}} \oint_{C_{n}} \frac{d z_{n}}{z_{n}} \oint_{C_{n-1}} \frac{d z_{n-1}}{z_{n-1}} \ldots \oint_{C_{1}} \frac{d z_{1}}{z_{1}} e\left(z_{1}\right) e\left(z_{2}\right) \ldots e\left(z_{n}\right) \otimes \\
f\left(z_{1}\right) f\left(z_{2}\right) \ldots f\left(z_{n}\right),
\end{gathered}
$$

and the contours $C_{k}$ enclose the origin, such that the points $z_{i}=q^{-2} z_{j}$ are outside the contours $C_{i}$ and the points $z_{i}=q^{2} z_{j}$ are inside the contours $C_{i}$ in the same sense as in Proposition 3.

The second comultiplication structure, which we again write for all $U_{q}^{(D)}(\widehat{\mathrm{g}})$ and drop an index of a simple root for $U_{q}^{(D)}\left(\widehat{\mathbf{s l}}_{\mathbf{2}}\right)$ looks as follows:

$$
\begin{gathered}
\Delta_{(I I)} e_{\alpha}(z)=e_{\alpha}(z) \otimes 1+K_{\alpha}^{+}\left(z q^{-c_{1} / 2}\right) \otimes e_{\alpha}\left(z q^{-c_{1}}\right), \\
\Delta_{(I I)} f_{\alpha}(z)=1 \otimes f_{\alpha}(z)+e_{\alpha}\left(z q^{-c_{2}}\right) \otimes K_{\alpha}^{-}\left(z q^{-c_{2} / 2}\right), \\
\Delta_{(I I)} K_{\alpha}^{+}(z)=K_{\alpha}^{+}\left(z q^{c_{2} / 2}\right) \otimes K_{\alpha}^{+}\left(z q^{-c_{1} / 2}\right), \\
\Delta_{(I I)} K_{\alpha}^{-}(z)=K_{\alpha}^{-}\left(z q^{-c_{2} / 2}\right) \otimes K_{\alpha}^{-}\left(z q^{c_{1} / 2}\right),
\end{gathered}
$$

is connected to the first one by a simple twist over "Cartan" generators:

$$
\Delta_{(I I)}=\mathcal{K}^{-1} \Delta_{(I)}^{\prime} \mathcal{K}
$$

From (3.37) it follows that the universal $R$-matrix for the comultiplication (II) has a form:

$$
\mathcal{R}_{(I I)}=\overline{\mathcal{R}}^{21} \mathcal{K},
$$

and

$$
\left(\mathcal{R}_{(I I)}^{21}\right)^{-1}=\left(\mathcal{K}^{21}\right)^{-1} \overline{\mathcal{R}}^{-1}
$$

where the entries of (3.38) and of (3.39) are given by (3.5), (3.6), (3.9), (3.10) and by (3.20), (3.32). 


\section{Properties of ordered $q$-exponential integrals}

The main feature of the tensor $f(z) \otimes e(z)$ which essentially implies the cocycle properties of the factor $\overline{\mathcal{R}}$ of the universal $R$-matrix for $U_{q}^{(D)}\left(\widehat{\mathbf{s l}}_{\mathbf{2}}\right)$ is that the function of two variables $G\left(z_{1}, z_{2}\right)=$ $f\left(z_{1}\right) f\left(z_{2}\right) \otimes e\left(z_{1}\right) e\left(z_{2}\right)$ is meromorphic in $\left(\mathbf{C}^{*}\right)^{2}$ and has two simple poles at $z_{1}=q^{ \pm 2} z_{2}$, the sum of whose residues is zero. Indeed, under assumption $|q|<1$ the analytical continuation of the function $G\left(z_{1}, z_{2}\right)$ from the region $\left|z_{1}\right|>\left|q^{-2} z_{2}\right|$ into the region $\left|q^{2} z_{2}\right|<\left|z_{1}\right|<\left|q^{-2} z_{2}\right|$ is given as

$$
G^{\prime}\left(z_{1}, z_{2}\right)=\frac{q^{-2} z_{1}-z_{2}}{z_{1}-q^{-2} z_{2}} f\left(z_{2}\right) f\left(z_{1}\right) \otimes e\left(z_{1}\right) e\left(z_{2}\right),
$$

and the analytical continuation of the function $G^{\prime}\left(z_{1}, z_{2}\right)$ from the region $\left|q^{2} z_{2}\right|<\left|z_{1}\right|<\left|q^{-2} z_{2}\right|$ to the region $\left|z_{1}\right|<\left|q^{2} z_{2}\right|$ is

$$
G^{\prime \prime}\left(z_{1}, z_{2}\right)=\frac{q^{-2} z_{1}-z_{2}}{z_{1}-q^{-2} z_{2}} \frac{q^{2} z_{1}-z_{2}}{z_{1}-q^{2} z_{2}} f\left(z_{2}\right) f\left(z_{1}\right) \otimes e\left(z_{2}\right) e\left(z_{1}\right)=f\left(z_{2}\right) f\left(z_{1}\right) \otimes e\left(z_{2}\right) e\left(z_{1}\right) .
$$

From (4.1) we see that integral of $G\left(z_{1}, z_{2}\right)$ over $z_{1}$ in a region $\left|z_{1}\right|>>\left|z_{2}\right|$ is the same as in a region $\left|z_{1}\right|<<\left|z_{2}\right|$, which means that the residues at the points $z_{1}=q^{ \pm 2} z_{2}$ cancel. Putting in a base the rule (4.1) of analytical continuation, we formulate in this section an abstract definition of $q$-exponential integrals and prove their main algebraical properties.

Let $x(z)$ be operator valued function of complex variable $z$, satisfying the following conditions:

(i) For any integer $n$ the products $x\left(z_{1}\right) x\left(z_{2}\right) \cdots x\left(z_{n}\right)$ define an analytical function in a region $\left|z_{1}\right|>>\left|z_{2}\right|>>\ldots>>\left|z_{n}\right|>0$ which analytical continuation is a meromorphic function in $\left(\mathbf{C}^{*}\right)^{n}$ with only singularities being the simple poles on shifted diagonals $z_{i}=q z_{j}, i \neq j$.

(ii) the analytical continuation of the product $x\left(z_{1}\right) x\left(z_{2}\right) \cdots x\left(z_{n}\right)$ is a symmetric function in

$$
X_{n}=\left(\mathbf{C}^{*}\right)^{n} \backslash \bigcup_{i \neq j}\left\{z_{i}=q z_{j}\right\}
$$

in other words, the commutativity

$$
x\left(z_{1}\right) x\left(z_{2}\right)=x\left(z_{2}\right) x\left(z_{1}\right)
$$

takes place in a sense of analytical continuations in $X_{n}$

For brevity we call such a function as almost commutative function with two poles. For any almost commutative function $x(z)$ with two poles we define ordered $q$-exponential integrals as follows:

$$
\begin{gathered}
\mathcal{P} \overrightarrow{\exp }_{q}\left(\frac{1}{2 \pi i} \oint x(z) \frac{d z}{z}\right)=1+\sum_{n>0} \frac{1}{n !(2 \pi i)^{n}} \oint_{C_{1}} \frac{d z_{1}}{z_{1}} \cdots \oint_{C_{n}} \frac{d z_{n}}{z_{n}} x\left(z_{1}\right) x\left(z_{2}\right) \cdots x\left(z_{n}\right), \\
\mathcal{P} \overrightarrow{\exp }_{q^{-1}}\left(\frac{1}{2 \pi i} \oint x(z) \frac{d z}{z}\right)=1+\sum_{n>0} \frac{1}{n !(2 \pi i)^{n}} \oint_{C^{\prime}{ }_{1}} \frac{d z_{1}}{z_{1}} \cdots \oint_{C^{\prime}{ }_{n}} \frac{d z_{n}}{z_{n}} x\left(z_{1}\right) x\left(z_{2}\right) \cdots x\left(z_{n}\right),
\end{gathered}
$$

where the contours $C_{i}$ and $C^{\prime}{ }_{i}$ enclose the origin and for the contours $C_{i}$ the points $z_{i}=q z_{j}$ are outside the contour while the points $z_{i}=q^{-1} z_{j}$ are inside; for the contours $C^{\prime}{ }_{i}$ the points $z_{i}=q z_{j}$ are inside the contour while the points $z_{i}=q^{-1} z_{j}$ are outside. We do not discuss here the convergence of the rhs of (4.3), (4.4) and treate them as formal series. 
We say also that two almost commutative functions $x_{1}(z)$ and $x_{2}(w)$ with two poles $q$ commute and denote this as $[x(z), y(w)]_{q}=0$ if

(iii) For any integer $n$ and for any sequence $\iota=i_{1}, i_{2}, \ldots, i_{n}$, where $i_{k}=1,2$ the products $x_{i_{1}}\left(z_{i_{1}, 1}\right) x_{i_{2}}\left(z_{i_{2}, 2}\right) \cdots x_{i_{n}}\left(z_{i_{1}, n}\right)$ are analytical functions in a region $\left|z_{i_{1}, 1}\right|>>\left|z_{i_{2}, 2}\right|>>\ldots>>$ $\left|z_{i_{n}, n}\right|>0$ which analytical continuation is a meromorphic function in $\left(\mathbf{C}^{*}\right)^{n}$ with only singularities being the simple poles on shifted diagonals $z_{1, k}=q z_{1, l}, k \neq l, z_{2, k}=q z_{2, l}, k \neq l$ and $z_{1, k}=q z_{2, l}$

(iv) the analytical continuation of the product $x_{i_{1}}\left(z_{i_{1}, 1}\right) x_{i_{2}}\left(z_{i_{2}, 2}\right) \cdots x_{i_{n}}\left(z_{i_{1}, n}\right)$ is a symmetric function in

$$
X_{\iota}=\left(\mathbf{C}^{*}\right)^{n} \backslash\left(\bigcup_{i, k, l: k \neq l}\left\{z_{i, k}=q z_{i, l}\right\} \cup_{k, l}\left\{z_{1, k}=q z_{2, l}\right\}\right),
$$

where the action of the symmetric group is defined as

$$
\sigma x_{i_{1}}\left(z_{i_{1}, 1}\right) \cdots x_{i_{n}}\left(z_{i_{1}, n}\right)=x_{i_{\sigma(1)}}\left(z_{i_{\sigma(1)}, \sigma(1)}\right) \cdots x_{i_{\sigma(n)}}\left(z_{i_{\sigma(n)}, \sigma(n)}\right) .
$$

In other words, the commutativity

$$
x_{1}(z) x_{2}(w)=x_{2}(w) x_{1}(z)
$$

takes place in a sense of analytical continuations in $X_{\iota}$.

One can see that for any two almost commutative functions $x(z)$ and $y(w)$, which $q$ commute, their sum $x(z)+y(z)$ is also almost commutative with two poles, i.e., it satisfies the conditions (i) and (ii). For two $q$-commuting functions $x(z)$ and $y(w)$ we denote also by $: x(q z) y(z):$ the residue

$$
: x(q z) y(z):=\frac{1}{2 \pi i} \oint_{w \text { around } q z} x(w) y(z) \frac{d w}{w} .
$$

The following proposition is a straightforward corollary of the definitions above.

Proposition 5 (a) For any almost commutative function $x(z)$ with two poles:

$$
\left(\mathcal{P} \overrightarrow{\exp }_{q}\left(\frac{1}{2 \pi i} \oint x(z) \frac{d z}{z}\right)\right)^{-1}=\mathcal{P} \overrightarrow{\exp }_{q^{-1}}\left(-\frac{1}{2 \pi i} \oint x(z) \frac{d z}{z}\right)
$$

(b) If two almost commutative functions $x(z)$ and $y(z) q$-commute, $[x(z), y(w)]_{q}=0$, then

$$
\mathcal{P} \overrightarrow{\exp }_{q}\left(\frac{1}{2 \pi i} \oint x(z)+y(z) \frac{d z}{z}\right)=\mathcal{P} \overrightarrow{\exp }_{q}\left(\frac{1}{2 \pi i} \oint y(z) \frac{d z}{z}\right) \mathcal{P} \overrightarrow{\exp }_{q}\left(\frac{1}{2 \pi i} \oint x(z) \frac{d z}{z}\right)
$$

(c) For two almost commutative functions

$$
\begin{gathered}
\mathcal{P} \overrightarrow{\exp }_{q}\left(\frac{1}{2 \pi i} \oint x(z) \frac{d z}{z}\right) \mathcal{P} \overrightarrow{\exp }_{q}\left(\frac{1}{2 \pi i} \oint y(z) \frac{d z}{z}\right)= \\
\mathcal{P} \overrightarrow{\exp }_{q}\left(\frac{1}{2 \pi i} \oint(x(z)+y(z)+: x(q z) y(z):) \frac{d z}{z}\right)
\end{gathered}
$$

It is not difficult to see that the statements of Proposition 3 and of Proposition 4 give another proof of the inversion property (4.6) for almost commutative function $f(z) \otimes e(z)$. The addition property (4.7) is equivalent in this sense to the properties

$$
(\Delta \otimes i d) \mathcal{R}=\mathcal{R}^{13} \mathcal{R}^{23}, \quad(i d \otimes \Delta) \mathcal{R}=\mathcal{R}^{13} \mathcal{R}^{12}
$$


of the universal $R$-matrix for $U_{q}^{(D)}\left(\widehat{\mathbf{s l}}_{\mathbf{2}}\right)$ and one could also consider (4.7) as the corollary of Propositions 3 and 4.

There is also an analog of H'Adamard identity for ordered $q$-exponential integrals. In order to write it down let us define the $q$-commutator operation

$$
\begin{aligned}
& {\left[\frac{1}{2 \pi i} \oint x(z) \frac{d z}{z}, \frac{1}{(2 \pi i)^{n}} \oint_{C_{1}} \frac{d w_{1}}{w_{1}} \cdots \oint_{C_{n}} \frac{d w_{n}}{w_{n}} A\left(w_{1}, \ldots, w_{n}\right)\right]_{q}=} \\
& =a d_{q} \frac{1}{2 \pi i} \oint x(z) \frac{d z}{z}\left(\frac{1}{(2 \pi i)^{n}} \oint_{C_{1}} \frac{d w_{1}}{w_{1}} \ldots \oint_{C_{n}} \frac{d w_{n}}{w_{n}} A\left(w_{1}, \ldots, w_{n}\right)\right)
\end{aligned}
$$

as

$$
\begin{gathered}
\frac{1}{(2 \pi i)^{n+1}}\left(\oint_{C_{1}} \frac{d w_{1}}{w_{1}} \cdots \oint_{C_{n}} \frac{d w_{n}}{w_{n}} \oint_{C} \frac{d z}{z} x(z) A\left(w_{1}, \ldots, w_{n}\right)-\right. \\
\left.-\oint_{C_{1}} \frac{d w_{1}}{w_{1}} \cdots \oint_{C_{n}} \frac{d w_{n}}{w_{n}} \oint_{C^{\prime}} \frac{d z}{z} A\left(w_{1}, \ldots, w_{n}\right) x(z)\right),
\end{gathered}
$$

where the contours $C$ and $C^{\prime}$ enclose the origin and the points $z=q w_{j}$ are outside the contour $C$ and inside the contour $C^{\prime}$ while the points $z=q^{-1} w_{j}$ are inside the contour $C$ and outside the contour $C^{\prime}$.

Proposition 6 For any almost commutative function $x(z)$ and any operator $A$ the following identity of formal series takes place:

$$
\mathcal{P} \overrightarrow{\exp }_{q}\left(\frac{1}{2 \pi i} \oint x(z) \frac{d z}{z}\right) A\left(\mathcal{P} \overrightarrow{\exp }_{q}\left(\frac{1}{2 \pi i} \oint x(z) \frac{d z}{z}\right)\right)^{-1}=A+\sum_{n>0} \frac{1}{n !} a d_{q}^{n} \frac{1}{2 \pi i} \oint x(z) \frac{d z}{z}(A) .
$$

It is also proved by direct calculations where the idintities between the sums of $q$-numbers are replaced by the identities between different contours integrals.

\section{$5 \quad$ A Hopf structure of $W_{q}(\widehat{\mathbf{g}}) \times U_{q}^{(D)}(\widehat{\mathbf{g}})$}

The semidirect product $W_{q}(\widehat{\mathbf{g}}) \times U_{q}^{(D)}(\widehat{\mathrm{g}})$ can be equiped with a structure of a Hopf algebra in the same manner as it was done for quntized envelopping algebras of Kac- Moody Lie algebras, see, e.g. [KR.

Let us recover an index of a simple root $\alpha$ in previous section and denote the tensor $\overline{\mathcal{R}}$ given in (3.7), (3.19) as $\overline{\mathcal{R}}_{\alpha}$ and if it come from the fields $e_{\alpha}(u)$ and of $f_{\alpha}(u)$. We claim the following

Proposition 7 The semidirect product $W_{q}(\widehat{\mathbf{g}}) \times U_{q}^{(D)}(\widehat{\mathbf{g}})$ is a Hopf algebra with respect to the comultiplication structure which is:

$$
\begin{gathered}
\Delta(x)=\Delta_{(I)}(x) \quad \text { for } x \in U_{q}^{(D)}(\widehat{\mathbf{g}}), \\
\Delta\left(T_{\alpha}\right)=\left(T_{\alpha} \otimes T_{\alpha}\right) \overline{\mathcal{R}}_{\alpha}, \quad \Delta\left(T_{\alpha}^{-1}\right)=\overline{\mathcal{R}}_{\alpha}^{-1}\left(T_{\alpha}^{-1} \otimes T_{\alpha}^{-1}\right), \\
\Delta\left(P_{\lambda}\right)=P_{\lambda} \otimes P_{\lambda} .
\end{gathered}
$$


By means of the ordered exponentials of composed root currents we can construct the universal $R$-matrix for arbitrary $U_{q}^{(D)}(\widehat{\mathrm{g}})$ in simple laced case. Let us fix the reduced decomposition of the longest element $w_{0}$ of the Weyl group of Lie algebra $\mathbf{g}$ of the rank $r$

$$
w_{0}=s_{\alpha_{i_{1}}} s_{\alpha_{i_{2}}} \cdots s_{\alpha_{i_{N}}}
$$

and let $\check{e}_{\gamma_{1}}(u), \check{e}_{\gamma_{2}}(u), \ldots, \check{e}_{\gamma_{N}}(u)$ and $\check{f}_{\gamma_{1}}(u), \check{f}_{\gamma_{2}}(u), \ldots, \check{f}_{\gamma_{N}}(u)$ be the composed root currents given by the prescription:

$$
\begin{aligned}
\check{e}_{\gamma_{N}}(u)=e_{\alpha_{i_{N}}}(u), & \check{f}_{\gamma_{N}}(u)=f_{\alpha_{i_{n}}}(u), \\
\check{e}_{\gamma_{N-1}}(u)=T_{\alpha_{i_{N}}}^{-1} e_{\alpha_{i_{N-1}}}(u), & \check{f}_{\gamma_{N-1}}(u)=T_{\alpha_{i_{N}}}^{-1} f_{\alpha_{i_{N-1}}}(u) \\
\check{e}_{\gamma_{N-2}}(u)=T_{\alpha_{i_{N}}}^{-1} T_{\alpha_{i_{N-1}}}^{-1} e_{\alpha_{i_{N-2}}}(u), & \check{f}_{\gamma_{N-2}}(u)=T_{\alpha_{i_{N}}}^{-1} T_{\alpha_{i_{N-1}}}^{-1} f_{\alpha_{i_{N-2}}}(u)
\end{aligned}
$$

and so on. Let also $\overline{\mathcal{R}}_{\gamma_{i}}$ be a tensor $\overline{\mathcal{R}}$ constructed from the composed fields $\check{e}_{\gamma_{i}}(u)$ and $\check{f}_{\gamma_{i}}(u)$ and

$$
\mathcal{K}=q^{-t} q^{\frac{-c \otimes d-d \otimes c}{2}} \prod_{n>0} \exp \left(-n\left(q-q^{-1}\right) \sum_{i, j=1}^{r} d_{i, j}^{(n)} a_{i, n} \otimes a_{j,-n}\right) q^{\frac{-c \otimes d-d \otimes c}{2}}
$$

where $t=\sum h_{i} \otimes h^{i}$ is an invariant tensor in tensor square of Cartan subalgebra $\mathbf{h} \otimes \mathbf{h}$ and $d_{i, j}^{(n)}$ is an inverse matrix to

$$
b_{i, j}^{(n)}=\left[n\left(\alpha_{i}, \alpha_{j}\right)\right]_{q}
$$

We claim that

Proposition 8 The tensor

$$
\mathcal{R}=\mathcal{K} \vec{\prod}_{1 \leq i \leq N} \overline{\mathcal{R}}_{\gamma_{i}}
$$

is the universal $R$-matrix for the algebra $U_{q}^{(D)}(\widehat{\mathbf{g}})$ with comultiplication $\Delta_{(I)}$.

The logic of the proofs of Propositions 5 and 6 is standard.. The role of the tensor $\mathcal{K}$ from (5.2) is the same as of $q^{t}$ in a Kac-Moody case: one can check directly, that it satisfies the condition (3.37). This condition reduces the proof of Propositions to the check of certain cocycle conditions on ordered $q$-exponential integrals. The new input is algebraical properties of $q$-exponential integrals which are the generalizations of the properties of usual $q$-exponential functions, (see, e.g., [KT1]) and are equivalent to the cocycle conditions. Note also, that in the formula (5.3) we can freely choose the presentation of the factors $\overline{\mathcal{R}}_{\gamma_{i}}$ either in a form of infinite product or in an integral form. The integral form is more convenient for the proof of propositions. Still, the infinite product variant of (5.3) gives a presentation of the universal $R$-matrix for $U_{q}^{(D)}(\widehat{\mathrm{g}})$, different from [KT3] if rank greater then one.

\section{The Yangian version}

It is not difficult to rewrite the whole story for the double of the Yangian. Let us choose here for definiteness the presentaion of the double of the Yangian $\mathcal{A}_{\hbar}(\widehat{\mathbf{g}})^{(D)}$ in Fourier integrals KLP where its generating functions are given by the prescriptions

$$
e_{\alpha_{i}}(u)=\int_{-\infty}^{+\infty} d \lambda e^{-i \lambda u} \hat{e}_{\alpha_{i}, \lambda}, \quad f_{\alpha_{i}}(u)=\int_{-\infty}^{+\infty} d \lambda e^{-i \lambda u} \hat{f}_{\alpha_{i}, \lambda}
$$




$$
K_{\alpha_{i}}^{ \pm}(u)=\exp \hbar \int_{0}^{ \pm \infty} d \lambda e^{-i \lambda u} \hat{a}_{i, \lambda}
$$

and satisfy the additive version of the relations $(1.3)-(1.13)$ :

$$
\begin{gathered}
(u-v+i \hbar(\alpha, \beta) / 2) e_{\alpha}(u) e_{\beta}(v)=e_{\beta}(v) e_{\alpha}(u)(u-v-i \hbar(\alpha, \beta) / 2), \\
(u-v-i \hbar(\alpha, \beta) / 2) f_{\alpha}(u) f_{\beta}(v)=f_{\beta}(v) f_{\alpha}(u)(u-v+i \hbar(\alpha, \beta) / 2), \\
\frac{\left(u-v+\frac{i \hbar(\alpha, \beta)}{2}-\frac{i \hbar c}{4}\right)}{\left(u-v-\frac{i \hbar(\alpha, \beta)}{2}-\frac{i \hbar c}{4}\right)} K_{\alpha}^{+}(u) e_{\beta}(v)=e_{\beta}(v) K_{\alpha}^{+}(u), \\
K_{\alpha}^{-}(u) e_{\beta}(v)=\frac{\left(u-v-\frac{i \hbar(\alpha, \beta)}{2}+\frac{i \hbar c}{4}\right)}{\left(u-v+\frac{i \hbar(\alpha, \beta)}{2}+\frac{i \hbar c}{4}\right)} e_{\beta}(v) K_{\alpha}^{-}(u), \\
\frac{\left(u-v-\frac{i \hbar(\alpha, \beta)}{2}+\frac{i \hbar c}{4}\right)}{\left(u-v+\frac{i \hbar(\alpha, \beta)}{2}+\frac{i \hbar c}{4}\right)} K_{\alpha}^{+}(u) f_{\beta}(v)=f_{\beta}(v) K_{\alpha}^{+}(u), \\
K_{\alpha}^{-}(u) f_{\beta}(v)=\frac{\left(u-v+\frac{i \hbar(\alpha, \beta)}{2}-\frac{i \hbar c}{4}\right)}{\left(u-v-\frac{i \hbar(\alpha, \beta)}{2}-\frac{i \hbar c}{4}\right)} f_{\beta}(v) K_{\alpha}^{-}(u), \\
\frac{\left(u-v+\frac{i \hbar(\alpha, \beta)}{2}-\frac{i \hbar c}{2}\right)\left(u-v-\frac{i \hbar(\alpha, \beta)}{2}+\frac{i \hbar c}{2}\right)}{\left(u-v+\frac{i \hbar(\alpha, \beta)}{2}+\frac{i \hbar c}{2}\right)\left(u-v-\frac{i \hbar(\alpha, \beta)}{2}-\frac{i \hbar c}{2}\right)} K_{\alpha}^{+}(u) K_{\beta}^{-}(v)=K_{\beta}^{-}(v) K_{\alpha}^{+}(u), \\
K_{\alpha}^{ \pm}(u) K_{\beta}^{ \pm}(v)=K_{\beta}^{ \pm}(v) K_{\alpha}^{ \pm}(u) \\
{\left[e_{\alpha}(u), f_{\beta}(v)\right]=\frac{i \delta_{\alpha, \beta}}{\hbar}\left[\delta\left(u-v+\frac{i c \hbar}{2}\right) K_{\alpha}^{+}\left(u+\frac{i c \hbar}{4}\right)-\delta\left(u-v-\frac{i c \hbar}{2}\right) K_{\alpha}^{-}\left(v+\frac{i c \hbar}{4}\right)\right],}
\end{gathered}
$$

and the Serre relations

$$
\begin{aligned}
& e_{\alpha}\left(u_{1}\right) e_{\alpha}\left(u_{2}\right) e_{\beta}(v)-2 e_{\alpha}\left(u_{1}\right) e_{\beta}(v) e_{\alpha}\left(u_{2}\right)+e_{\beta}(v) e_{\alpha}\left(u_{1}\right) e_{\alpha}\left(u_{2}\right)+u_{1} \leftrightarrow u_{2}=0 \\
& f_{\alpha}\left(u_{1}\right) f_{\alpha}\left(u_{2}\right) f_{\beta}(v)-2 f_{\alpha}\left(u_{1}\right) f_{\beta}(v) f_{\alpha}\left(u_{2}\right)+f_{\beta}(v) f_{\alpha}\left(u_{1}\right) f_{\alpha}\left(u_{2}\right)+u_{1} \leftrightarrow u_{2}=0
\end{aligned}
$$

for any $\alpha, \beta,(\alpha, \beta)=-1$. Here $\delta$ function is defined as

$$
\delta(u-v)=\lim _{\epsilon \rightarrow 0}\left[\frac{1}{u-v-i \epsilon}-\frac{1}{u-v+i \epsilon}\right]=i \int_{-\infty}^{\infty} d \lambda \mathrm{e}^{-i \lambda(u-v)} .
$$

The highest weight representations are characterized by the property that

$$
\hat{e}_{\alpha, \lambda} v=\hat{f}_{\alpha, \lambda} v=\hat{a}_{\alpha, \lambda} v=0
$$

for any vector $v$ in the representation space for $\lambda$ big enough. It means, in particular, that operator valued functions $e_{\alpha}(u) e_{\beta}(v)$ are analytical in a region $\operatorname{Im} u>>\operatorname{Im} v$, and the relations (1.3) allow to define the analytical continuation of these functions into a region $\operatorname{Im} u<\operatorname{Im}(v-$ $i \hbar(\alpha, \beta) / 2)$ as

$$
e_{\alpha}(u) e_{\beta}(v)=\frac{\left(u-v-\frac{i \hbar(\alpha, \beta)}{2}\right)}{\left(u-v+\frac{i \hbar(\alpha, \beta)}{2}\right)} e_{\beta}(v) e_{\alpha}(u) .
$$

The definition of composed root vectors has a form:

$$
e_{\alpha+\beta}(v)=: e_{\alpha}(v-i \hbar / 2) e_{\beta}(v-i \hbar):, \quad f_{\alpha+\beta}(v)=: f_{\beta}(v-i \hbar) f_{\alpha}(v-i \hbar / 2):,
$$




$$
\check{e}_{\alpha+\beta}(v)=: e_{\beta}(v+i \hbar) e_{\alpha}(v+i \hbar / 2):, \quad \check{f}_{\alpha+\beta}(v)=: f_{\alpha}(v+i \hbar / 2) f_{\beta}(v+i \hbar):,
$$

that is

$$
\begin{aligned}
& e_{\alpha+\beta}(v)=\frac{1}{2 \pi i}\left(\int_{C_{+i \infty}} e_{\alpha}(u) e_{\beta}(v-i \hbar) d u-\int_{C_{-i \infty}} \frac{\left(u-v+\frac{3 i \hbar}{2}\right)}{\left(u-v+\frac{i \hbar}{2}\right)} e_{\beta}(v-i \hbar) e_{\alpha}(u) d u\right), \\
& f_{\alpha+\beta}(v)=\frac{1}{2 \pi i}\left(-\int_{C_{-i \infty}} f_{\beta}(v-i \hbar) f_{\alpha}(u) d u+\int_{C_{+i \infty}} \frac{\left(u-v+\frac{3 i \hbar}{2}\right)}{\left(u-v+\frac{i \hbar}{2}\right)} f_{\alpha}(u) f_{\beta}(v-i \hbar) d u\right),
\end{aligned}
$$

where $C_{+i \infty}$ is a contour going from $-\infty$ to $+\infty$ in such a way that the points $u=v-i \hbar / 2$ and $u=+i \infty$ are on the different sides of the contour and $C_{-i \infty}$ is a contour going from $-\infty$ to $+\infty$ in such a way that the points $u=v-i \hbar / 2$ and $u=-i \infty$ are on the different sides of the contour;

$$
\begin{gathered}
\check{e}_{\alpha+\beta}(v)=\frac{1}{2 \pi i}\left(-\int_{C^{\prime}-i \infty} e_{\beta}(v+i \hbar) e_{\alpha}(u) d u+\int_{C^{\prime}+i \infty} \frac{\left(u-v-\frac{3 i \hbar}{2}\right)}{\left(u-v-\frac{i \hbar}{2}\right)} e_{\alpha}(u) e_{\beta}(v+i \hbar) d u\right), \\
\check{f}_{\alpha+\beta}(v)=\frac{1}{2 \pi i}\left(\int_{C^{\prime}+i \infty} f_{\alpha}(u) f_{\beta}(v+i \hbar) d u-\int_{C^{\prime}-i \infty} \frac{\left(u-v-\frac{3 i \hbar}{2}\right)}{\left(u-v-\frac{i \hbar}{2}\right)} f_{\beta}(v+i \hbar) f_{\alpha}(u) d u\right),
\end{gathered}
$$

where $C^{\prime}+i \infty$ is a contour going from $+\infty$ to $-\infty$ in such a way that the points $u=v+i \hbar / 2$ and $u=+i \infty$ are on the different sides of the contour and $C^{\prime}{ }_{-i \infty}$ is a contour going from $+\infty$ to $-\infty$ in such a way that the points $u=v+i \hbar / 2$ and $u=-i \infty$ are on the different sides of the contour.

The Serre relations are equivalent to

$$
\begin{aligned}
e_{\alpha}(u) e_{\alpha+\beta}(v)=\frac{u-v-i \hbar / 2}{u-v+i \hbar / 2} e_{\alpha+\beta}(v) e_{\alpha}(u), & \operatorname{Im} u<\operatorname{Im}(v-i \hbar / 2), \\
e_{\alpha+\beta}(u) e_{\beta}(v)=\frac{u-v-2 i \hbar}{u-v-i \hbar} e_{\beta}(v) e_{\alpha+\beta}(u), & \operatorname{Im} u<\operatorname{Im}(v+i \hbar) \\
f_{\beta}(u) f_{\alpha+\beta}(v)=\frac{u-v+2 i \hbar}{u-v+\hbar} f_{\alpha+\beta}(v) f_{\alpha}(u), & \operatorname{Im} u<\operatorname{Im}(v-i \hbar) \\
f_{\alpha+\beta}(u) f_{\alpha}(v)=\frac{u-v+i \hbar / 2}{u-v-i \hbar / 2} f_{\alpha}(v) f_{\alpha+\beta}(u), & \operatorname{Im} u<\operatorname{Im}(v+i \hbar / 2)
\end{aligned}
$$

and the Weyl group automorphisms have a form

$$
\begin{gathered}
T_{\alpha} e_{\alpha}(u)=f_{\alpha}\left(u+\frac{i \hbar c}{2}\right) K_{\alpha}^{+}\left(u+\frac{i \hbar c}{4}\right)^{-1}, \\
T_{\alpha} f_{\alpha}(u)=K_{\alpha}^{-}\left(u+\frac{i \hbar c}{4}\right)^{-1} e_{\alpha}\left(u+\frac{i \hbar c}{2}\right), \\
T_{\alpha} K_{\alpha}^{ \pm}(u)=K_{\alpha}^{ \pm}(u)^{-1}, \quad T_{\alpha} K_{\beta}^{ \pm}(u)=K_{\alpha}^{ \pm}\left(u-\frac{i \hbar}{2}\right) K_{\beta}^{ \pm}(u-i \hbar), \quad(\alpha, \beta)=-1, \\
T_{\alpha} e_{\beta}(u)=e_{\alpha+\beta}(u), \quad T_{\alpha} f_{\beta}(u)=f_{\alpha+\beta}(u) \cdot \quad(\alpha, \beta)=-1, \\
T_{\alpha}^{-1} e_{\alpha}(u)=K_{\alpha}^{-}\left(u-\frac{i \hbar c}{4}\right)^{-1} f_{\alpha}\left(u-\frac{i \hbar c}{2}\right), \\
T_{\alpha}^{-1} f_{\alpha}(u)=e_{\alpha}\left(u-\frac{i \hbar c}{2}\right) K_{\alpha}^{+}\left(u-\frac{i \hbar c}{4}\right)^{-1},
\end{gathered}
$$




$$
\begin{gathered}
T_{\alpha}^{-1} K_{\alpha}^{ \pm}(u)=K_{\alpha}^{ \pm}(u)^{-1}, \quad T_{\alpha}^{-1} K_{\beta}^{ \pm}(u)=K_{\alpha}^{ \pm}\left(u+\frac{i \hbar}{2}\right) K_{\beta}^{ \pm}(u+i \hbar), \quad(\alpha, \beta)=-1, \\
T_{\alpha}^{-1} e_{\beta}(u)=\check{e}_{\alpha+\beta}(u), \quad T_{\alpha}^{-1} f_{\beta}(u)=\check{f}_{\alpha+\beta}(u) \quad(\alpha, \beta)=-1, \\
P_{\varepsilon \omega_{\alpha}} e_{\alpha}(u)=e^{i \varepsilon u} e_{\alpha}(u), \quad P_{\varepsilon \omega_{\alpha}} f_{\alpha}(u)=e^{-i \varepsilon u} f_{\alpha}(u), \quad \varepsilon \in \mathbf{R} \\
P_{\varepsilon \omega_{\alpha}} K_{\alpha}^{ \pm}(u)=e^{ \pm \varepsilon \hbar / 2} K_{\alpha}^{ \pm}(u) .
\end{gathered}
$$

Here the weight lattice $Q$ is replaced by its real form $Q_{\mathbf{R}}=Q \otimes_{\mathbf{Z}} \mathbf{R}$. All the statements of the first sections remain valid in the Yangian case.

The universal $R$-matrix for $\mathcal{A}_{\hbar}\left(\widehat{\mathbf{s l}}_{\mathbf{2}}\right)^{(D)}$ with the comultiplication rule

$$
\begin{gathered}
\Delta e_{\alpha}(u)=e_{\alpha}(u) \otimes 1+K_{\alpha}^{-}\left(u-i c_{1} \hbar / 4\right) \otimes e_{\alpha}\left(u-i c_{1} \hbar / 4\right), \\
\Delta f_{\alpha}(u)=1 \otimes f_{\alpha}(u)+f_{\alpha}\left(u-i c_{2} \hbar / 4\right) \otimes K_{\alpha}^{+}\left(u-i c_{2} \hbar / 4\right), \\
\Delta K_{\alpha}^{+}(u)=K_{\alpha}^{+}\left(u-i c_{2} \hbar / 4\right) \otimes K_{\alpha}^{+}\left(u+i c_{1} \hbar / 4\right), \\
\Delta K_{\alpha}^{-}(u)=K_{\alpha}^{-}\left(u+i c_{2} \hbar / 4\right) \otimes K_{\alpha}^{-}\left(u-i c_{1} \hbar / 4\right)
\end{gathered}
$$

can be presented as

$$
\mathcal{R}=\mathcal{K} \overline{\mathcal{R}}
$$

where $\left(d=\frac{d}{d u}\right)$

$$
\begin{gathered}
\mathcal{K}=e^{i \hbar(d \otimes c+c \otimes d) / 4} \exp \left(-\int_{0}^{+\infty} d \lambda \frac{\hbar^{2} \lambda}{2 \operatorname{sh} \hbar \lambda} \hat{a}_{\lambda} \otimes \hat{a}_{-\lambda}\right) e^{i \hbar(d \otimes c+c \otimes d) / 4} \\
\overline{\mathcal{R}}=\vec{P} \exp \left(-\hbar \int_{-\infty}^{+\infty} d \lambda \hat{f}_{-\lambda} \otimes \hat{e}_{\lambda}\right) .
\end{gathered}
$$

or, equivalently,

$$
\overline{\mathcal{R}}=\mathcal{P} \overrightarrow{\exp }_{i \hbar}\left(\frac{\hbar}{2 \pi} \int_{-\infty}^{+\infty} f(u) \otimes e(u) d u\right)
$$

where

$$
\begin{gathered}
\mathcal{P} \overrightarrow{\exp }_{i \hbar}\left(\frac{\hbar}{2 \pi} \int_{-\infty}^{+\infty} f(u) \otimes e(u) d u\right)=1+ \\
\sum_{n>0} \frac{\hbar^{n}}{n !(2 \pi)^{n}} \int_{C_{n}} d u_{n} \int_{C_{n-1}} d u_{n-1} \ldots \oint_{C_{1}} d u_{1} f\left(u_{1}\right) f\left(u_{2}\right) \ldots f\left(u_{n}\right) \otimes \\
e\left(u_{1}\right) e\left(u_{2}\right) \ldots e\left(u_{n}\right),
\end{gathered}
$$

and the contours $C_{k}$ are going from $-\infty$ to $+\infty$ in such a way that the points $u_{k}=u_{j}+i \hbar$ and the point $-i \infty$ are on on the one side of the contour $C_{k}$ while the points $u_{k}=u_{j}-i \hbar$ and the point $+i \infty$ are on the other side of the contour. The arguments of the section 4 can be repeated here and they give the universal $R$-matrix for $\mathcal{A}_{\hbar}(\widehat{\mathbf{g}})^{(D)}$ for any simple laced $\mathbf{g}$.

For the usual presentation of the Yangian double $\widehat{D Y}(\mathbf{g})^{(D)}$ by Laurent series (see, e.g., [KLP]), we use instead of the marked points $\pm \infty$ zero and infinity, as in the case of quantum affine algebra. The formulas are the same except (6.4) and (6.5) where the lattice appear again and the definition of affine shifts is more close to the case of quantum affine algebras. In an analogous manner we can treate the face type elliptic algebras and the elliptic algebras in scaling limit with Drinfeld's type comultiplication. We will observe it a separate note.

\section{Acknowlegements}


This work was done when the second author visited RIMS. He use the opportunity to appreciate the Institute and Prof. T. Miwa for the kind hospitality. The authors are grateful to Profs. V. Bazhanov, L.D. Faddeev, B. Feigin, S. Kharchev, S. Pakuliak and F. Smirnov for the discussions. We thak also Profs. S.Kharchev and S.Pakuliak for the help in checking the key formulas. S.Kh. was supported by INTAS grant 93-10183, RFBR grant 98-01-00303 and grant 96-15-96455 for support of scientific schools.

\section{Appendix A. The braid group relation}

Let us examine the identity

$$
T_{\alpha} T_{\beta} T_{\alpha}\left(e_{\beta}(z)\right)=T_{\beta} T_{\alpha} T_{\beta}\left(e_{\beta}(z)\right)
$$

for adjacent roots $\alpha$ and $\beta$. From the definition of automorphisms and by simple use of (1.5) we get

$$
\begin{gathered}
T_{\beta} T_{\alpha}\left(e_{\beta}(z)\right) \cdot K_{\beta}^{+}\left(q^{2-c / 2} z\right)^{-1}= \\
\frac{1}{(2 \pi i)^{2}}\left[\oint_{C_{\infty}} \frac{d u}{u}\left(\oint_{C_{\infty}} \frac{d v}{v} e_{\beta}(v) e_{\alpha}\left(q^{2} u\right)-\oint_{C_{0}} \frac{d v}{v} \frac{q^{-1} v-q^{2} u}{v-q u} e_{\alpha}\left(q^{2} u\right) e_{\beta}(v)\right) f_{\beta}\left(q^{2-c} z\right)-\right. \\
\left.\oint_{C_{0}} \frac{d u}{u} f_{\beta}\left(q^{2-c} z\right)\left(\oint_{C_{\infty}} \frac{d v}{v} e_{\beta}(v) e_{\alpha}\left(q^{2} u\right)-\oint_{C_{0}} \frac{d v}{v} \frac{q^{-1} v-q^{2} u}{v-q u} e_{\alpha}\left(q^{2} u\right) e_{\beta}(v)\right)\right]= \\
\frac{1}{(2 \pi i)^{2}\left(q-q^{-1}\right)} \oint_{C_{\infty}} \frac{d u}{u}\left(\oint_{C_{\infty}}-\oint_{C_{0}}\right) \frac{d v}{v} \frac{q^{-1} v-q^{2} u}{v-q u} \delta\left(v / q^{2} z\right) e_{\alpha}\left(q^{2} u\right) K_{\beta}^{+}\left(q^{2-c / 2} z\right)- \\
\frac{1}{(2 \pi i)^{2}\left(q-q^{-1}\right)} \oint_{C_{\infty}} \frac{d u}{u}\left(\oint_{C_{\infty}}-\oint_{C_{0}}\right) \frac{d v}{v} \delta\left(v / q^{2-2 c} z\right) K_{\beta}^{-}\left(v q^{c / 2}\right) e_{\alpha}\left(q^{2} u\right)
\end{gathered}
$$

Here the contour $C_{\infty}$ for the variable $u$ means that it enclose the origin and the point $u=q z$ is inside while for the contour $C_{0}$ this point is outside. For the contour $C_{\infty}$ for the variable $v$ the point $v=q u$ is inside while for $C_{0}$ it is inside.

The integration over $v$ cancel $\delta$ function and evaluate the integrand in a point $v=q^{2} z$ for the first integral and in a point $v=q^{2-2 c} z$ for the second. Then the integration over $u$ is a taking a residue in a point $u=q z$ for the first term (since the rest is normal ordered) and is zero for the second term. As a result, we have

$$
T_{\alpha} T_{\beta}\left(e_{\beta}(z)\right)=q e_{\alpha}\left(q^{3} z\right)
$$

which gives

$$
T_{\alpha} T_{\beta} T_{\alpha}\left(e_{\beta}(z)\right)=q f_{\alpha}\left(q^{3-c} z\right) K_{\alpha}^{+}\left(q^{3-c / 2} z\right)^{-1}
$$

Analogously,

$$
T_{\alpha} T_{\beta}\left(f_{\beta}(z)\right)=q f_{\alpha}\left(q^{3} z\right)
$$

and

$$
\begin{gathered}
T_{\beta} T_{\alpha} T_{\beta}\left(e_{\beta}(z)\right)=T_{\beta} T_{\alpha}\left(f_{\beta}\left(q^{-c z}\right) K_{\beta}^{+}\left(q^{-c / 2} z\right)^{-1}\right)= \\
q f_{\alpha}\left(q^{3-c} z\right) K_{\alpha}^{+}\left(q^{3-c / 2} z\right)^{-1} .
\end{gathered}
$$

which proves (A.1). 


\section{Appendix B. Quadratic terms of $\overline{\mathcal{R}}$}

We want to compare the quadratic terms of the expressions (3.32) and (3.10). Let us for simplisity of notations restrict ourselves to degree $(0,0)$ terms and drop the common factor $\left(q-q^{-1}\right)^{-2}$.

As we noted before, we can compare only those series in generators of the algebra, which are given in normal ordered form with respect to the grading index. The ordering can be given by the following rules, which are equivalent to defining relations (1.3), (1.4) (see [KT3]):

$$
\begin{gathered}
e_{n+2 k} e_{n}=q^{2} e_{n} e_{n+2 k}+\left(q^{4}-1\right)\left(e_{n+1} e_{n+2 k-1}+q^{2} e_{n+2} e_{n+2 k-2}+\ldots\right. \\
\left.+q^{2(k-2)} e_{n+k-1} e_{n+k+1}\right)+\left(q^{2}-1\right) q^{2(k-1)} e_{n+k}^{2} \quad \text { for any } k>0 \\
e_{n+2 k+1} e_{n}=q^{2} e_{n} e_{n+2 k+1}+\left(q^{4}-1\right)\left(e_{n+1} e_{n+2 k}+q^{2} e_{n+2} e_{n+2 k-1}+\ldots\right. \\
\left.+q^{2(k-1)} e_{n+k} e_{n+k+1}\right) \quad \text { for any } k \geq 0 \\
f_{n+2 k} f_{n}=q^{-2} f_{n} f_{n+2 k}+\left(q^{-4}-1\right)\left(f_{n+1} f_{n+2 k-1}+q^{-2} f_{n+2} f_{n+2 k-2}+\ldots\right. \\
\left.+q^{-2(k-2)} f_{n+k-1} f_{n+k+1}\right)+\left(q^{-2}-1\right) q^{-2(k-1)} f_{n+k}^{2} \quad \text { for any } k>0, \\
f_{n+2 k+1} f_{n}=q^{-2} f_{n} f_{n+2 k+1}+\left(q^{-4}-1\right)\left(f_{n+1} f_{n+2 k}+q^{-2} f_{n+2} f_{n+2 k-1}+\ldots\right. \\
\left.+q^{-2(k-1)} f_{n+k} f_{n+k+1}\right) \quad \text { for any } k \geq 0 .
\end{gathered}
$$

The calculation of the integral

$$
\oint_{C_{1}} \frac{d z_{1}}{z_{1}} \oint_{C_{2}} \frac{d z_{2}}{z_{2}} e\left(z_{1}\right) e\left(z_{2}\right) \otimes f\left(z_{1}\right) f\left(z_{2}\right)
$$

for the contours, enclosing the origin and inside a region $\left|q^{2} z_{2}\right|<\left|z_{1}\right|<\left|q^{-2} z_{2}\right|$ can be performed as follows: we take first the integral in the region of analyticity $\left|z_{1}\right|>>\left|z_{2}\right|$ and substruct an integral over $z_{2}$ of a residue (over $z_{1}$ ) in a point $z_{1}=q^{-2} z_{2}$.

The regular term gives an expression

$$
\sum_{n, m \in \mathbf{Z}} e_{-m} e_{-n} \otimes f_{m} f_{n}
$$

The corresponding normal ordered expression for its $(0,0)$ component has a form:

$$
\begin{gathered}
e_{0}^{2} \otimes f_{0}^{2}+\left(1-q^{-2}\right) \sum_{k>0} q^{2 k} e_{0}^{2} \otimes f_{-k} f_{k}+\left(1-q^{2}\right) \sum_{k>0} q^{-2 k} e_{-k} e_{k} \otimes f_{0}^{2}+\left(q^{2}+q^{-2}\right) \sum_{k>0} e_{-k} e_{k} \otimes f_{-k} f_{k}+ \\
\quad\left(q^{2}-q^{-2}\right) \sum_{k, l>0, l<k} q^{2 l} e_{-k+l} e_{k-l} \otimes f_{-k} f_{k}+\left(q^{-2}-q^{2}\right) \sum_{k, l>0, l<k} q^{-2 l} e_{-k} e_{k} \otimes f_{-k+l} f_{k-l} . \quad \text { (B.6) }
\end{gathered}
$$

The residue over $z_{1}$ at a point $z_{1}=q^{-2} z_{2}$ comes from the simple pole of $f\left(z_{1}\right) f\left(z_{2}\right)$ at this point. So, we should take the residue of $f\left(z_{1}\right) f\left(z_{2}\right)$ and multiply by the evaluation $e\left(q^{-2} z_{2}\right) e\left(z_{2}\right)$. The first expression can be given as a difference of two integrals:

$$
: f\left(q^{-2} z\right) f(z):=
$$




$$
\frac{1}{2 \pi i} \oint_{C_{1}} f\left(z_{1}\right) f(z) \frac{d z_{1}}{z_{1}}-\frac{1}{2 \pi i} \oint_{C_{2}} \frac{z_{1}-q^{2} z_{2}}{q^{2} z_{1}-z_{2}} f(z) f\left(z_{1}\right) \frac{d z_{1}}{z_{1}}
$$

where both contours enclose the origin and the point $z_{1}=q^{-2} z$ is inside the first contour and outside the second. Each of them can be derived by integration of corresponding Laurent series. This gives

$$
\begin{aligned}
& : f\left(q^{-2} z\right) f(z):=\left(q^{-2}-q^{2}\right) \sum_{n \in \mathbf{Z}} u^{-2 n-1} q^{2 n+2}\left(\sum_{k \geq 0} q^{2 k} f_{n-k} f_{n+k+1}\right)+ \\
& \left(q^{-2}-q^{2}\right) \sum_{n \in \mathbf{Z}} u^{-2 n} q^{2 n}\left(\left(1-q^{2}\right) f_{n}^{2}+\left(q^{-2}-q^{2}\right) \sum_{k>0} q^{2 k} f_{n-k} f_{n+k}\right)
\end{aligned}
$$

The evaluation of $e\left(q^{-2} z\right) e(z)$ assumes the direct application of (B.1), (B.2). In an assumption $|q|<1$ it gives an analogous expression:

$$
\begin{gathered}
e\left(q^{-2} z\right) e(z)=\sum_{n \in \mathbf{Z}} u^{-2 n-1} q^{2 n+2}\left(\sum_{k \geq 0} q^{-2 k} e_{n-k} e_{n+k+1}\right)+ \\
\sum_{n \in \mathbf{Z}} u^{-2 n} q^{2 n}\left(\frac{e_{n}^{2}}{1+q^{2}}+\sum_{k>0} q^{-2 k} e_{n-k} e_{n+k}\right)
\end{gathered}
$$

For $|q|>1$ one should first perfom an analytical continuation (since in (B.8) we summed up the geometric progressions over $q)$, that is to use instead of $e(u) e(v)$ its analitycal continuation $\left(u-q^{2} v\right) /\left(q^{2} u-v\right) e(v) e(u)$ and then do the same. The answer will coincide with $(\overline{\mathrm{B} .8)})$. The integral of the residue reduces to the integration of a tensor product of power series (B.7) and (B.8). Its (0,0)- degree component has a form:

$$
\begin{gathered}
\frac{\left(1-q^{2}\right)}{1+q^{2}} e_{0}^{2} \otimes f_{0}^{2}+\left(1-q^{2}\right) \sum_{k>0} q^{-2 k} e_{-k} e_{k} \otimes f_{0}^{2}+ \\
\left(q^{-2}-1\right) \sum_{k>0} q^{2 k} e_{0}^{2} \otimes f_{-k} f_{k}+\left(q^{-2}-q^{2}\right) \sum_{k, p>0} q^{2(k-p)} e_{-p} e_{p} \otimes f_{-k} f_{k}
\end{gathered}
$$

The half of the difference $(\overline{B .6})$ and $(\mathrm{B.9})$ is

$$
\begin{gathered}
R_{2}^{0}=\frac{1}{1+q^{-2}} e_{0}^{2} \otimes f_{0}^{2}+\left(1-q^{-2}\right) \sum_{k>0} q^{2 k} e_{0}^{2} \otimes f_{-k} f_{k}+q^{2} \sum_{k>0} e_{-k} e_{k} \otimes f_{-k} f_{k}+ \\
\left(q^{2}-q^{-2}\right) \sum_{k, l>0, l<k} q^{2 l} e_{-k+l} e_{k-l} \otimes f_{-k} f_{k} .
\end{gathered}
$$

On the other hand, we can pick up the quadratic term of the infinite product (3.7) modulo factor $\left(q-q^{-1}\right)^{-2}$ :

$$
{R^{\prime}}_{2}^{0}=\sum_{\substack{n, m \in \mathbf{Z} \\ n<m}} e_{-n} e_{-m} \otimes f_{n} f_{m}+\frac{1}{1+q^{-2}} \sum_{n \in \mathbf{Z}} e_{-n}^{2} \otimes f_{n}^{2}
$$

Performing normal ordering in the first tensor component, we get the expression, which $(0,0)$ degree term coincides with $(\overline{B .10})$. 


\section{References}

[BLZ] Bazhanov,V., Lukyanov, S. and Zamolodchikov, A. Integrable Structure of Conformal Field Theory, Quantum KdV Theory and Thermodynamic Bethe Ansatz. Commun. Math. Phys. 177 (1996) 381-404.

[Be] Beck, J. Braid group action and quantum affine algebras. Com. Math. Phys. 165 (1994), 555-568.

[DF] Ding, J., and Frenkel, I.B. Isomorphism of two realizations of quantum affine algebras. Comm. Math. Phys, 156 (1994), 277-300.

[DI] Ding, J., Iohara, K., Drinfeld comultiplucation and vertex operators. J. of Geom. and Physics 23, (1997), 1-13.

[DK] Ding, J., Khoroshkin, S., On the FRTS approach to quantized current algebras. preprint math-Q/9804??? (1998).

[Dr1] Drinfeld, V.G. Quantum groups. Proc. ICM-86 (Berkely USA) vol.1, 798-820. Amer. Math. Soc. (1987).

[Dr2] Drinfeld, V.G. A new realization of Yangians and quantized affine algebras. Soviet Math. Dokl. 32 (1988), 212-216.

[Dr3] Drinfeld, V.G. A new realization of Yangians and quantized affine algebras. FTINT Preprint 30-86 (1986).

[ER] Enriquez, B., Rubtsov, V. Quasi-Hopf algebras associated with $s l(2)$ and complex curves, Preprint q-alg/9608005, 1996.

[FV] Faddeev, L.D and Volkov, A. Yu., Abelian current algebra and the Virasoro algebra on the lattice. Phys. Lett. B315 (1993), 311-318.

[KT1] Khoroshkin, S.M., and Tolstoy, V.N., Universal R-matrix for quantized (super)algebras, Commun. Math. Phys. 141, 1991, 599-617.

[KT2] Khoroshkin, S.M., and Tolstoy, V.N., On Drinfeld realization of quantum affine algebras, Journal of Geometry and Physics, 11, 1993, 101-108.

[KT3] Khoroshkin, S.M., and Tolstoy, V.N., Twisting of quantum (super)algebras. Connection of Drinfeld's and Cartan-Weyl realizations for quantum affine algebras, Max Planck Institute Preprint MPI/94-23, hep-th/9404036, 1994, 1-25.

[KLP] S. Khoroshkin, D. Lebedev, S. Pakuliak, Yangian algebras and classical Riemann problems, Preprint q-alg/9712057 (1997)

[KR] Kirillov, A.N., and Reshetikhin, N.Yu. q-Weyl group and a multiplicative formula for universal R-matrices. Comm. Math. Phys. 134 (1990), 421-431.

[L] Lusztig, G. Canonical bases arising from quantized enveloping algebras. J. Amer. Math. Soc. 3 (1990), 447-498.

[V] Volkov, A. Yu., q-combinatorics and quantum integrability. Preprint q-alg/9702007 (1997). 